\title{
Demonstration of a Neural Circuit Critical for Imprinting Behavior in Chicks
}

\author{
Tomoharu Nakamori, ${ }^{1,3}$ Katsushige Sato, ${ }^{2,4}$ Yasuro Atoji, ${ }^{5}$ Tomoyuki Kanamatsu, ${ }^{6}$ Kohichi Tanaka, ${ }^{1}$ \\ and Hiroko Ohki-Hamazaki ${ }^{1,3,7}$ \\ ${ }^{1}$ Laboratory of Molecular Neuroscience, School of Biomedical Science and Medical Research Institute and 2Department of Physiology and Cell Biology, \\ Faculty of Medicine, Graduate School, Tokyo Medical and Dental University, Bunkyo-ku, Tokyo 113-8519, Japan, ${ }^{3}$ Division of Biology, College of Liberal \\ Arts and Sciences, Kitasato University, Sagamihara, Kanagawa 228-8555, Japan, ${ }^{4}$ Department of Health and Nutrition Sciences, Faculty of Human Health, \\ Komazawa Women's University, Inagi-shi, Tokyo 206-8511, Japan, ${ }^{5}$ Laboratory of Veterinary Anatomy, Faculty of Applied Biological Sciences, Gifu \\ University, Gifu 501-1193, Japan, ${ }^{6}$ Department of Environmental Engineering for Symbiosis, Faculty of Engineering, Soka University, Hachioji, Tokyo \\ 192-8577, Japan, and ${ }^{7}$ Recognition and Formation, Precursory Research for Embryonic Science and Technology, Japan Science and Technology Agency, \\ Kawaguchi, Saitama 332-0012, Japan
}

Imprinting behavior in birds is elicited by visual and/or auditory cues. It has been demonstrated previously that visual cues are recognized and processed in the visual Wulst (VW), and imprinting memory is stored in the intermediate medial mesopallium (IMM) of the telencephalon. Alteration of neural responses in these two regions according to imprinting has been reported, yet direct evidence of the neural circuit linking these two regions is lacking. Thus, it remains unclear how memory is formed and expressed in this circuit. Here, we present anatomical as well as physiological evidence of the neural circuit connecting the VW and IMM and show that imprinting training during the critical period strengthens and refines this circuit. A functional connection established by imprint training resulted in an imprinting behavior. After the closure of the critical period, training could not activate this circuit nor induce the imprinting behavior. Glutamatergic neurons in the ventroposterior region of the VW, the core region of the hyperpallium densocellulare (HDCo), sent their axons to the periventricular part of the HD, just dorsal and afferent to the IMM. We found that the HDCo is important in imprinting behavior. The refinement and/or enhancement of this neural circuit are attributed to increased activity of HDCo cells, and the activity depended on NR2B-containing NMDA receptors. These findings show a neural connection in the telencephalon in Aves and demonstrate that NR2B function is indispensable for the plasticity of HDCo cells, which are key mediators of imprinting.

\section{Introduction}

During infancy, children are highly sensitive to environmental stimuli and their neural network is easily shaped, which results in the acquisition of new functions and skills. The cellular and molecular basis of this plasticity is not yet fully understood because of the limited number of available animal models. In contrast, the neural plasticity of the sensory cortex during infancy has been studied extensively in animal subjects that are partially deprived of visual (Wiesel and Hubel, 1965; Antonini and Stryker, 1993; Antonini et al., 1999) or somatosensory input (Van der Loos and Woolsey, 1973; Woolsey and Wann, 1976). It has been shown that NMDA receptors (NMDARs) are one of the molecules implicated in cortical

\footnotetext{
Received July 8, 2009; revised Jan. 11, 2010; accepted Feb. 17, 2010.

This work was supported by Precursory Research for Embryonic Science and Technology of the Japan Science and Technology Agency, Grants-in-Aid from the Ministry of Education, Culture, Sports, Science, and Technology of Japan, and by the Sasakawa Scientific Research Grant from The Japan Science Society. We thank K. Wada and E. D. Jarvis for supplying the plasmids used for the in situ hybridization (NR1, NR2A, and NR2B), Y. Honda and I. Sugihara for instruction for tracer injection and detection, and the laboratory members of Tokyo Medical and Dental University and Kitasato University for their help and discussions.

Correspondence should be addressed to Hiroko Ohki-Hamazaki, Division of Biology, College of Liberal Arts and Sciences, Kitasato University, 1-15-1 Kitazato, Sagamihara, Kanagawa 228-8555, Japan. E-mail: hamazaki@ kitasato-u.ac.jp.

D0I:10.1523/JNEUROSCI.3532-09.2010

Copyright $\odot 2010$ the authors $\quad 0270-6474 / 10 / 304467-14 \$ 15.00 / 0$
}

map development and plasticity in the barrel cortex (Schlaggar et al., 1993; Iwasato et al., 2000), as well as in the visual cortex (Kleinschmidt et al., 1987; Bear et al., 1990; Roberts et al., 1998).

These studies have elucidated the mechanism of artificially induced plasticity in the two sensory systems, but we still do not know whether or not the plasticity that enables early learning and memory can be induced by the same mechanism. Therefore, with the aim of elucidating the mechanism of naturally occurring early learning, we performed a study using baby chickens. During the first few days after hatching, chicks show "imprinting behavior" (Lorenz, 1937). Previous studies have demonstrated that the visual Wulst (VW), bearing homologies to the mammalian visual cortex, and the intermediate medial mesopallium (IMM), which is partially similar to the mammalian associate cortex (Reiner et al., 2004), play crucial roles in the process of imprinting (Horn et al., 1979; Kohsaka et al., 1979; McCabe et al., 1981; Maekawa et al., 2006). We have shown recently that visual imprinting is accompanied by plastic changes in the VW that result in an increase of the region responding to the imprint stimulus as well as an increase in the number of cholecystokinin-positive neurons (Maekawa et al., 2006, 2007). The processed visual information necessary for eliciting imprinting behavior should be transmitted to the IMM, which serves as the memory-storage region. The VW is the center of the thalamofugal pathway in birds, and the inter- 
stitial nucleus of the hyperpallium apicale (IHA) in the VW receives visual input from the nucleus dorsolateralis anterior (DLA) of the thalamus (Karten et al., 1973; Watanabe et al., 1983), but the anatomical connection between the VW and IMM has not yet been clarified.

We postulate that the neural connection between the VW and IMM may be critical for visual imprinting. To test this hypothesis, we explored and identified the neural connections between the VW and IMM using neural tracers. We identified the ventroposterior part of the VW, the core region of the hyperpallium densocellulare (HDCo), as a key region that controls the activity of this circuit. The outcome of the training depended on the activity of the HDCo neurons bearing NR2B subunits of the NMDAR, and the enhanced activity of these cells may enable visual imprinting behavior.

\section{Materials and Methods}

Animals. Fertilized eggs from White Leghorn chickens (Akebono Farm) were incubated at $37.7^{\circ} \mathrm{C}$ under moderate moisture and quasi-constant darkness. After hatching, chicks were kept in groups in the same incubator [dark condition (d)]. The experimental protocols described in this paper were approved by the Institutional Animal Care and Use Committee of Tokyo Medical and Dental University.

Imprinting device. Visual imprinting was performed with a device described previously (Maekawa et al., 2006, 2007). Briefly, a running wheel connected to a custom-made computer system was used to record the chick's movements toward or away from the display (Muromachi Kikai). A liquid crystal monitor (15-inch Flex Scan L367, EIZO; Nanao) was placed on each side of the wheel, and an image was displayed on one of the two monitors. The images were generated by a visual stimulus generator system (VSG; Cambridge Research Systems). The square had 8.6$\mathrm{cm}$-long sides ( $24^{\circ}$ of the visual field), the circle was $8.6 \mathrm{~cm}$ in diameter, and each image bounced left and right horizontally on the screen at a rate of $7.3 \mathrm{~cm} / \mathrm{s}\left(20.9^{\circ} / \mathrm{s}\right)$. All colors had a luminance of $10.36 \mathrm{~cd} / \mathrm{m}^{2}$, and the Commission Internationale de l'Eclairage $x y$ chromaticity coordinates of the tested colors were as follows: red, 0.658 and 0.307 ; blue, 0.15 and 0.062 .

Training and evaluation of visual imprinting. Chicks were placed in the running wheel and exposed to a training image (a red square) presented on the left and right monitors (30 min for each side) or only on the left monitor for $30 \mathrm{~min}$. We confirmed that either protocol induced imprinting behavior. For the control training (c), chicks were put into the apparatus for $30 \mathrm{~min}$ or $1 \mathrm{~h}$ without any image on the screen. The duration of training was constant within the experiment. We trained chicks once at postnatal day 1 (P1) (24-48 h after hatching, TP1) unless otherwise specified. After the training, they were put back into the same incubator as before the training.

Unless otherwise specified, $24 \mathrm{~h}$ after the training, the chicks were put into the imprinting apparatus again. After a 5 min adaptation period (black monitor, no presentation of the image), the red square and the blue circle (see Figs. $5 F, 6,8$ ) were presented sequentially every 5 min on the left monitor. In the experiments described in Figures 4 and 9 and supplemental Fig. S4 (available at www.jneurosci.org as supplemental material), after a 5 min adaptation time, the red and blue squares were presented sequentially as above. After a 5 min adaptation time, only the red square was presented (see Fig. 7). The direction and number of wheel revolutions were recorded. We calculated the preference score (PS) (McCabe et al., 1982) using the following formula as an index of success of visual imprinting: PS = SUM (training image) $/$ SSUM (training image) + SUM (new image)\}, where SUM (training image) is the number of wheel revolutions toward the display during the presentation of the training stimulus, and SUM (new image) is the number of wheel revolutions toward the display during the presentation of the new image. The chicks that rotated the wheel $<22.5$ revolutions during the 15 min evaluation period were excluded from the analysis (Maekawa et al., 2007).

General histological methods. Chicks were anesthetized with diethylether and perfused with $4 \%$ paraformaldehyde (PFA). The whole brains were postfixed in the same fixative for $24 \mathrm{~h}$ at $4^{\circ} \mathrm{C}$, cryoprotected by immersing in $30 \%$ sucrose for $48 \mathrm{~h}$, embedded in the Tissue-Tek O.T.C. Compound (Sakura Finetek), and frozen in powdered dry ice. Using a cryostat (CM1900; Leica Microsystems), 30- to 50- $\mu \mathrm{m}$-thick sagittal sections were prepared. The immunostained sections were mounted on glass slides, dried, dehydrated, and coverslipped.

Tracer injection and detection. Anesthesia was induced in P1 or P7 chicks by the intraperitoneal injection of $2 \%$ 2,2,2-tribromoethanol (Nacalai Tesque) dissolved in saline. The chicks were placed in a stereotaxic frame (model 900; David Kopf Instruments), and an incision was made along the midline to expose the skull. A small region of the skull just above the planned injection site was bored with an electro microdrill (UC500; Urawa Minitor).

For anterograde tracing experiments, several parts of the VW, the HDCo and the periventricular region of the hyperpallium densocellulare (HDPe), were selected as injection areas. For each region, 100-300 $\mathrm{nl}$ of $10 \%$ biotinylated dextran amine (BDA) (List Biological Laboratories) in PBS was injected unilaterally with a glass micropipette (15-30 $\mu \mathrm{m}$ tip diameter) using a pressure system (Eppendorf FemtoJet Microinjector) (Honda and Ishizuka, 2004). After the survival period of $7 \mathrm{~d}$, chicks were deeply anesthetized and perfused with $4 \%$ PFA.

The sections were washed three times in $0.5 \%$ Triton X-100 in PBS (PBST) at room temperature for $30 \mathrm{~min}$, PBST containing $0.3 \% \mathrm{H}_{2} \mathrm{O}_{2}$ for $30 \mathrm{~min}, \mathrm{PBST}$ for $5 \mathrm{~min}$ three times, and 3\% normal goat serum including $0.5 \%$ Triton $\mathrm{X}-100$ (NGST) for $2 \mathrm{~h}$. Then, the sections were reacted with avidin-biotin complex (ABC Elite; Vector Laboratories) in 3\% NGST for $48 \mathrm{~h}$ at $4^{\circ} \mathrm{C}$. They were washed with PBS for $20 \mathrm{~min}$ three times, treated with $0.1 \% 3,3^{\prime}$-diaminobenzidine tetrahydrochloride (DAB) with $0.1 \%$ ammonium nickel (II) sulfate (Nacalai Tesque), and washed with $0.1 \mathrm{M}$ Tris- $\mathrm{HCl}$ buffer, $\mathrm{pH}$ 8.0, to stop the reaction (Sugihara and Shinoda, 2004).

For the retrograde tracing experiments, we injected 100-300 $\mathrm{nl}$ of $0.5 \%$ cholera toxin subunit B (CTb) (List Biological Laboratories) in PBS into several parts of the VW, HDCo, HDPe, and IMM in each P1 chick or in HDPe in each P7 chick. We used the same injection method as described in BDA injections. Goat anti-CTb $\operatorname{IgG}\left(1: 20,000,12 \mathrm{~h}\right.$ at $4^{\circ} \mathrm{C}$; List Biological Laboratories) was used for primary antibody, and biotinylated donkey anti-goat IgG (Jackson ImmunoResearch) was used for secondary antibody before the reaction with the avidin-biotin complex (ABC Elite; Vector Laboratories) (Luppi et al., 1990; Shibata et al., 2004).

Immunostaining with anti-cFos antibody. The sections were treated as described above, and rabbit anti-cFos antibody (1:3000; Santa Cruz Biotechnology) was applied to the sections for $12 \mathrm{~h}$ at $4^{\circ} \mathrm{C}$. After washing with PBST, the sections were reacted with a polymer reagent including peroxidase and goat anti-rabbit IgG antibody (Dako Envision kit/HRP) for $1 \mathrm{~h}$ at room temperature. Then, they were treated with $0.1 \% \mathrm{DAB}$ to visualize peroxidase and washed with $0.1 \mathrm{M}$ Tris- $\mathrm{HCl}$ buffer, $\mathrm{pH} 8.0$, to stop the reaction.

For double immunostaining with anti-CTb antibodies, the sections were washed with PBS for 60 min after DAB treatment and soaked in $0.1 \mathrm{M}$ glycine- $\mathrm{HCl}$ buffer, $\mathrm{pH}$ 2.2, for $30 \mathrm{~min}$ three times, PBS for $5 \mathrm{~min}$ three times, PBS containing $\mathrm{H}_{2} \mathrm{O}_{2}$ for $30 \mathrm{~min}$, PBS for 5 min three times, and anti-CTb antibody in $3 \%$ normal goat serum for $12 \mathrm{~h}$ at $4^{\circ} \mathrm{C}$. Then, the sections were treated as described above in the tracer injection and detection protocol.

In situ hybridization. In situ hybridization was performed with freefloating sections according to procedures described previously (Maekawa et al., 2007). The zebra finch NR1, NR2A, and NR2B probes were kindly provided by Dr. Wada (Duke University, Durham, NC) (Wada et al., 2004). Vesicular glutamate transporter 2 (vGlut2) and GAD65 probes were described previously (Maekawa et al., 2007). Control experiments were performed, and no specific signals were observed when the sections were processed with the digoxigenin-labeled sense RNA probes.

For the double staining of vGlut2 or GAD65 mRNA by in situ hybridization and $\mathrm{CTb}$ protein by immunohistochemistry, in situ hybridization was performed first, followed by immunohistochemistry.

DiI tracing. Whole brains from P1 chicks were fixed with $4 \%$ PFA at $4^{\circ} \mathrm{C}$. Crystals of DiI (D-282; Invitrogen) were inserted into the HDCo or 

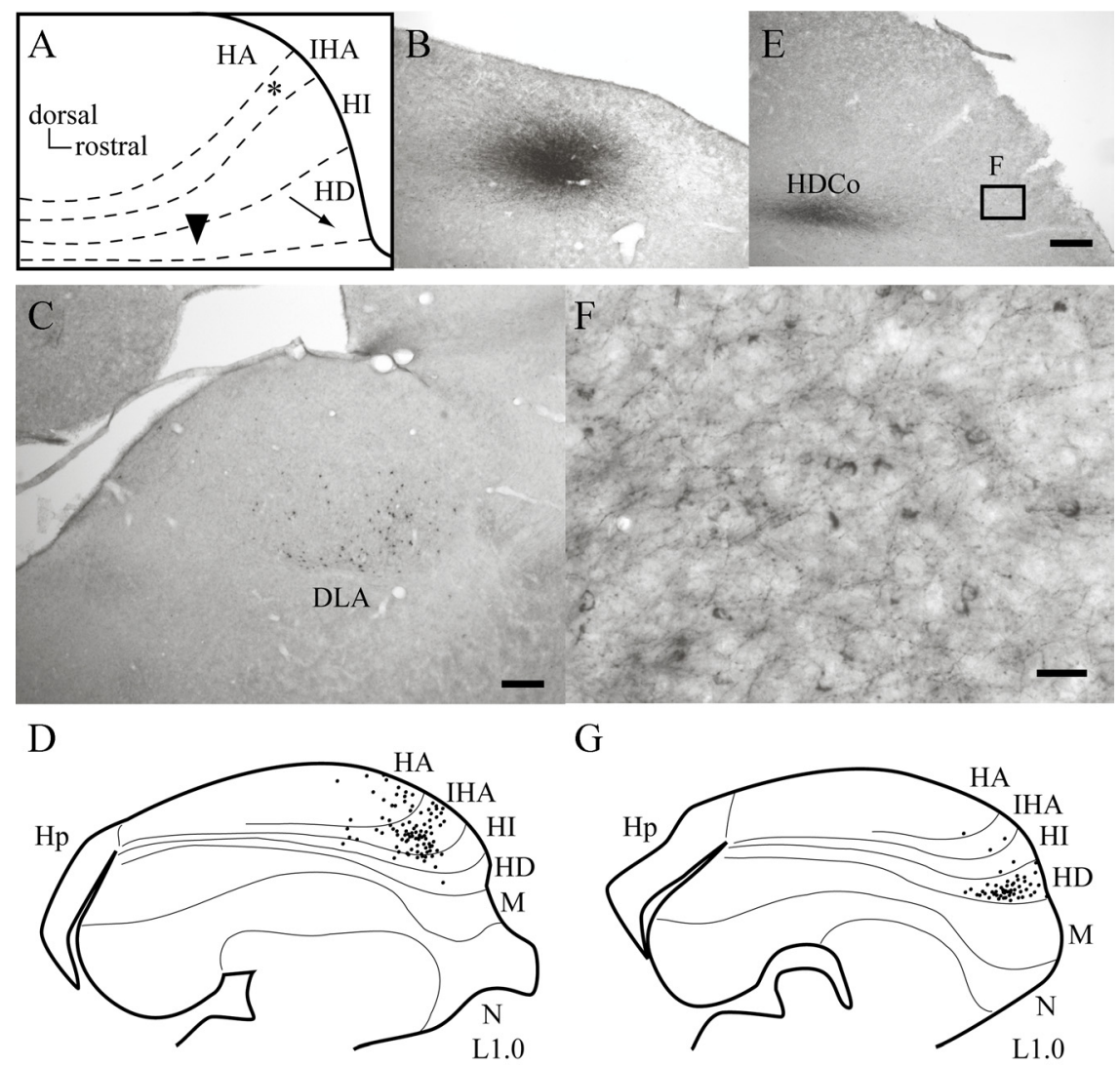

G

L1.0

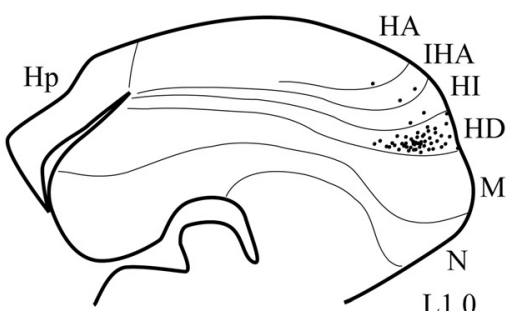

L1.0
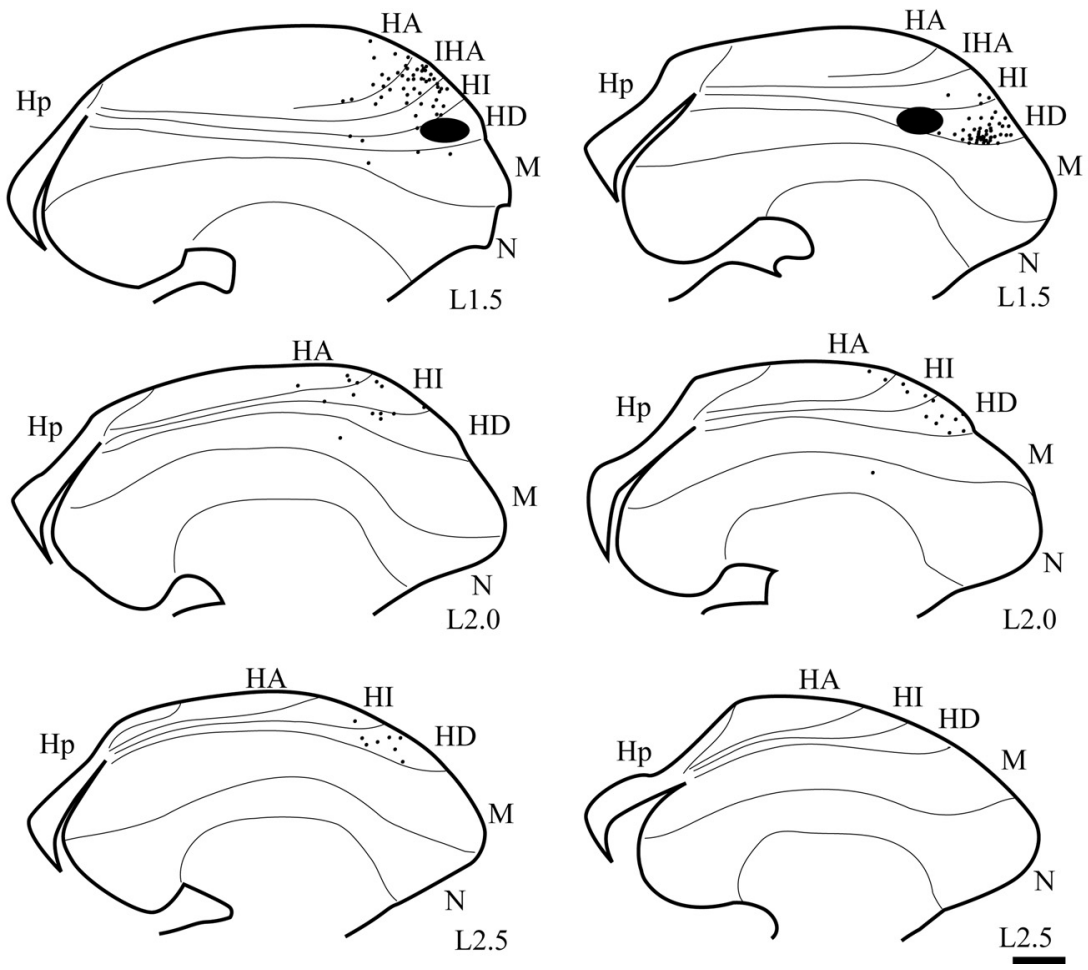

Figure 1. Analysis of neural connections in the VW using a retrograde tracer. $A$, Schematic representation of a sagittal section including the VW. The main structures (the HA, IHA, HI, and HD) and the (Tb injection sites (asterisk, arrow, and arrowhead) are indicated. $B, C, E, F$, Photomicrographs of CTb-labeled cells. $B$, CTb injection site in the IHA, which corresponds to the asterisk in $A$. $\boldsymbol{C}$, After the CTb injection shown in $\boldsymbol{B}(n=3)$, labeled cells were detected in the DLA of the thalamus. $\boldsymbol{D}$, A series of drawings spanning the mediolateral extent of the telencephalon, to illustrate schematically the (Tb injection site (solid black) in the HDR [corresponds to the arrow in $\boldsymbol{A}$ and (Tb-immunoreactive cell bodies (dots)] $(n=4)$. Hp, Hippocampus; M, mesopallium; N, nidopallium. $\boldsymbol{E}$, Injection of CTb in the HDCo (the arrowhead in $\boldsymbol{A})(n=5)$. $\boldsymbol{F}$, Enlarged photomicrograph of the box in $\boldsymbol{E}$. $\boldsymbol{G}$, A series of drawings of the telencephalon to illustrate the injection site (solid black) in the HDCo and the labeled cell bodies (dots). Scale bars: $\boldsymbol{C}, \boldsymbol{E}, 100 \mu \mathrm{m} ; \boldsymbol{F}, 25 \mu \mathrm{m} ; \boldsymbol{G}, 1 \mathrm{~mm}$.
HDPe. Brains were incubated in $4 \%$ PFA at $37^{\circ} \mathrm{C}$. Sagittal slices at $50 \mu \mathrm{m}$ thickness were cut with a microslicer (TDK-1500; Dousaka) and mounted. Images were acquired using a confocal fluorescence microscope (FV500; Olympus Optical) with software (FLUOVIEW; Olympus Optical).

Cell count. We used three chicks for the histological analysis in each condition and selected three sagittal sections per brain, which included the VW, hyperpallium apicale (HA), hyperpallium intercalatum (HI), and IMM.

Images were acquired under the constant exposure condition using a Leica (DMRA) microscope equipped with a DFC300FX digital camera and Leica Application Suite software. The light-microscopic images were transferred to a program (Adobe Illustrator 10.0; Adobe Systems) in which the brightness and contrast were adjusted to a fixed level.

To compare the number of positive cells between all conditions, we first determined the threshold level in 256-shade grayscale, which adequately reflected the positive cells. This threshold level was kept constant for all samples of the same staining. We measured the number of pixels in each $225 \times 300 \mu \mathrm{m}$ square of each region using Scion Image (Scion Corporation). Considering the size of the cells, particles that consisted of fewer than 10 pixels were not counted. We compared the number of positive cells counted by the experimenter with the number of pixels counted as described above and examined the correlation between these two values. From the results, we deduced that the number of positive cells corresponded to $1 / 100$ of the counted pixel number. When double staining was performed, we counted the single or double-positive cells manually (see Fig. 7D).

Microinjection into the $V W$. The NMDAR antagonist DL-2-amino-5-phosphonovaleric acid (APV) (200 $\mu \mathrm{M}, 2 \mu \mathrm{l}$; Sigma-Aldrich), the NR2B antagonist ifenprodil $(10 \mu \mathrm{M}, 2 \mu \mathrm{l}$; Sigma-Aldrich), or saline containing $0.1 \%$ Evans blue dye $(2 \mu \mathrm{l})$ was injected with a syringe (Hamilton Company) into P1 chicks. The chick's head was held in a horizontal position, and the injection was positioned $3 \mathrm{~mm}$ caudally from the posterior edge of the left eye, 2 $\mathrm{mm}$ laterally from the midline of the skull, and $2 \mathrm{~mm}$ depth from the skull surface. Five minutes after the injection, imprinting training was performed using a red square image. At P7, the imprinting performance was evaluated, and the chicks were used for optical imaging analysis.

Ibotenic acid (5 $\mu \mathrm{g} / \mu \mathrm{l}$; Sigma-Aldrich) or saline with $0.1 \%$ Evans blue dye was injected in the VW of $\mathrm{P} 0$ chicks by the same method. One day later, the imprinting training was performed. The imprinting behavior was evaluated $2 \mathrm{~h}$ after the training. After perfusing with $4 \%$ PFA, the brain was removed and treated as described above (see Histology). Sagittal sections at $50 \mu \mathrm{m}$ thickness were stained with $0.1 \%$ thionin.

Discrimination test. The P0 chicks were injected with ibotenic acid or saline in the HDCo as described above and placed in a cage $(20 \times$ 

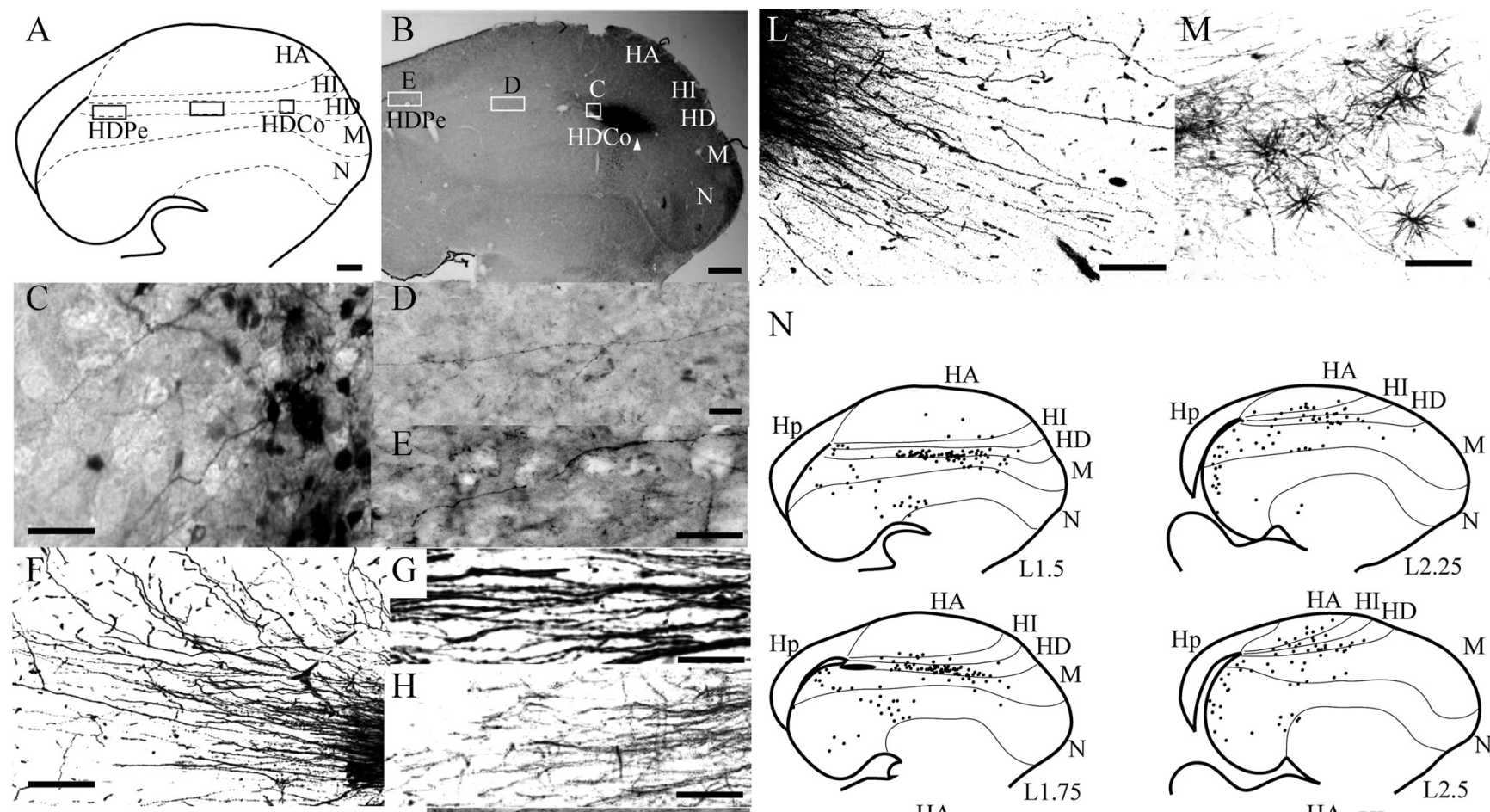

$\mathrm{D}$

$\mathrm{N}$
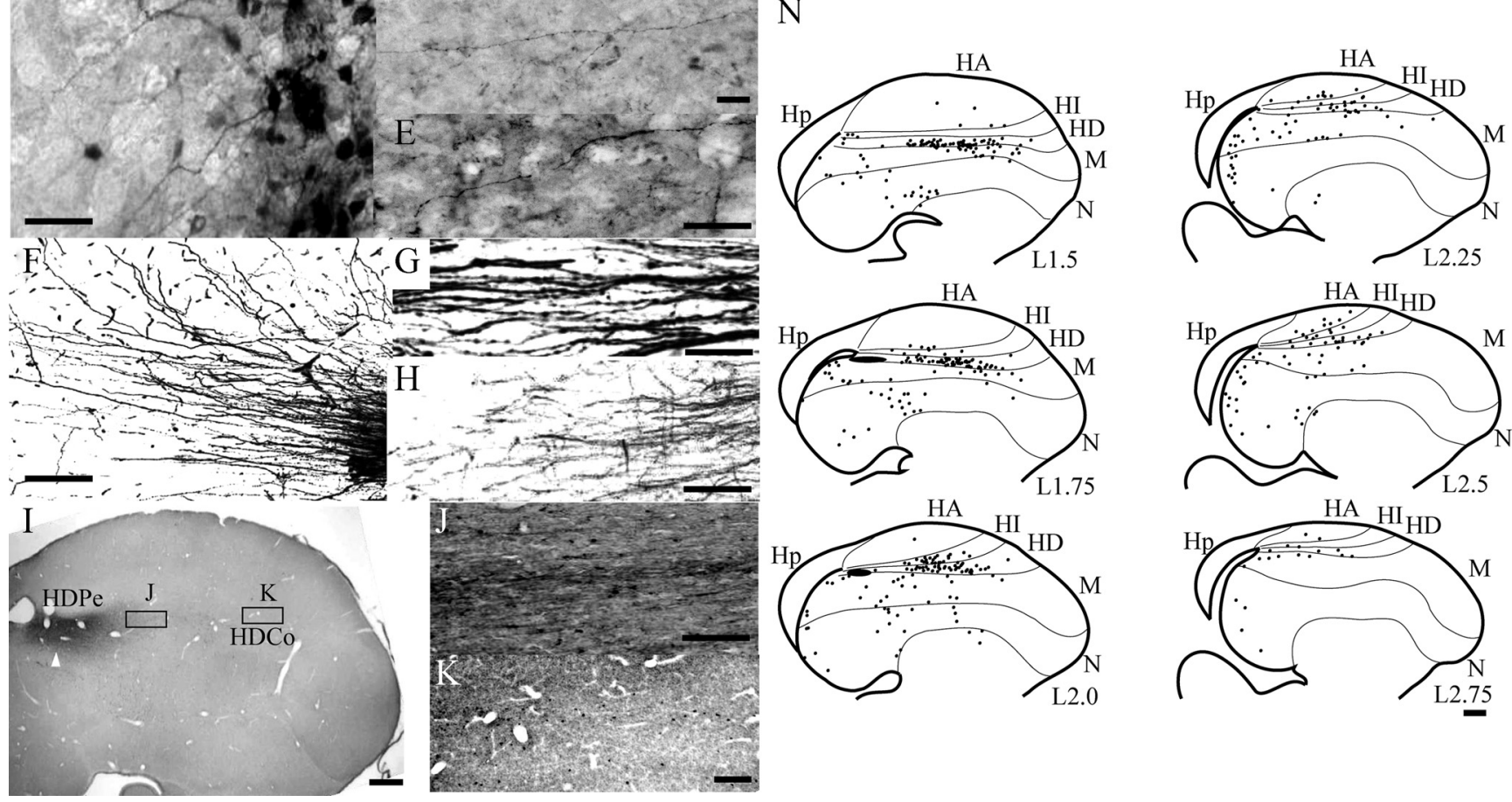

$\mathrm{L} 2.75$

Figure 2. Efferent projection of the HDCo neurons. $A$, Schema of a sagittal section of the chick telencephalon (L1.5), which depicts the related structures. $B$, Photomicrograph of the section injected with the anterograde neural tracer BDA in the $H D C 0$ (white arrowhead) $(n=5)$. C $-\boldsymbol{E}$, Enlargements of the corresponding boxes in $\boldsymbol{B}$. $\boldsymbol{F}-\boldsymbol{H}, \mathrm{A}$ Dil crystal was inserted into the HDCo, and the section was observed under fluorescence microscopy (each region corresponds to the boxes marked C $-E$ in $\boldsymbol{B}$, respectively) $(n=8)$. $I$, Photomicrograph of a sagittal section injected with CTb in the HDPe (white arrowhead) $(n=9) . J, K$, Enlargements of the corresponding regions of boxes J and $K$ in $I$. K represents the HDCo region. $L, M, A$ Dil crystal was inserted into the HDPe. Labeled fibers near the insertion site $(\boldsymbol{L})$ and labeled neurites and cell bodies in the $\mathrm{HDC}_{(}(\boldsymbol{M})(n=4) . \boldsymbol{N}$, A series of drawings spanning the mediolateral extent of the telencephalon to illustrate the $C T$ injection site (solid black) and (Tb-immunoreactive cell bodies (dots). Scale bars: $\boldsymbol{A}, \boldsymbol{B}, \boldsymbol{I}, \boldsymbol{N}, 1 \mathrm{~mm} ; \boldsymbol{C}, \boldsymbol{E}, \boldsymbol{G}, \boldsymbol{M}, 50 \mu \mathrm{m} ; \boldsymbol{D}, \boldsymbol{F}, \boldsymbol{H}, \boldsymbol{J}-\boldsymbol{L}, 100 \mu \mathrm{m}$. Hp, Hippocampus; M, mesopallium; N, nidopallium.

$30 \times 15 \mathrm{~cm})$ in the quasi-constant dark incubator until the discrimination test, which was performed the next day. We essentially used a protocol described previously (Gibbs and Summers, 2005), with slight modifications. A pair of chicks in the cage was placed in the room with a brightness of 80 lux, and each chick was presented sequentially with 1 min intervals, with one of the tips of a shiny metal bar (radius of $1 \mathrm{~mm}$; control test), red beads (radius of $2 \mathrm{~mm}$; red test) attached on the one end of the metal bar, and blue beads (radius of $2 \mathrm{~mm}$; blue test) similarly attached on the metal bar. The tip of the bar was placed $2-3 \mathrm{~cm}$ from the birds' beak, and the response of the chick was scored as the number of pecks during the $10 \mathrm{~s}$ presentation. One session of the discrimination test contained these three tests, and three sessions were performed with 10 min intervals. To obtain the scores of each chick for the control, red, and blue test, the average pecking numbers in the three sessions were calculated.

Slice preparation for optical recording. The details of the protocol for measuring electrical activity with multiple-site optical imaging techniques have been described previously (Orbach et al., 1985). Ringer's solution (in mm: $138 \mathrm{NaCl}, 5.4 \mathrm{KCl}, 1.8 \mathrm{CaCl}_{2}, 0.5 \mathrm{MgCl}_{2}$, and 10 Tris$\mathrm{HCl}, \mathrm{pH}$ 7.27) was cooled on ice with $\mathrm{O}_{2}$ bubbling. $\mathrm{P} 1, \mathrm{P} 4$, and $\mathrm{P} 7$ chicks were anesthetized with diethylether, and their brains were removed. Sagittal sections at $300 \mu \mathrm{m}$ thickness were cut with a microslicer (DTK3000W; Dousaka) in the cooled Ringer's solution. Then, the sections were kept in Ringer's solution at a temperature of $37^{\circ} \mathrm{C}$ under $\mathrm{O}_{2}$ aeration until the recording.

Staining with voltage-sensitive dye. Each slice was attached to the bottom of a plastic chamber with tungsten pins and maintained in Ringer's solution at $37^{\circ} \mathrm{C}$. The sections were stained for $10 \mathrm{~min}$ with a voltagesensitive merocyanine-rhodanine dye, NK2761 (0.04\% in Ringer's solution containing 1\% DMSO; Hayashibara Biochemical Laboratories) (Kamino et al., 1981; Salzberg et al., 1983; Obaid et al., 1985). Thereafter, the excess dye was washed out with dye-free Ringer's solution for $90 \mathrm{~min}$ to allow the slice to recover.

Electrical stimulation and optical imaging techniques. A bipolar tungsten microelectrode was used for the electrical stimulation. We put the microelectrode on a slice and applied an electric stimulus. The intensity and duration of stimulation were $600 \mu \mathrm{A}$ and $250 \mu \mathrm{s}$, respectively. Light was supplied from a $300 \mathrm{~W}$ tungsten-halogen lamp (type JC-24V/300W; Kondo Philips) and rendered quasi-monochromatic with a heat filter (32.5B-76; Olympus Optical) and an interference filter having its transmission maximum at $703 \pm 15 \mathrm{~nm}$ (Asahi Spectra). It was focused on the preparation by means of a bright-field condenser with a numerical aperture (NA) matched to that of the microscope objective (S plan Apo: $2 \times$, $0.08 \mathrm{NA} ; 10 \times, 0.4 \mathrm{NA})$. The optical signals were recorded at a sampling rate of $0.941 \mathrm{~ms}$ per frame using a photodiode array (PDA) with 464 channels arranged hexagonally (Miyakawa et al., 2003; Momose-Sato et 

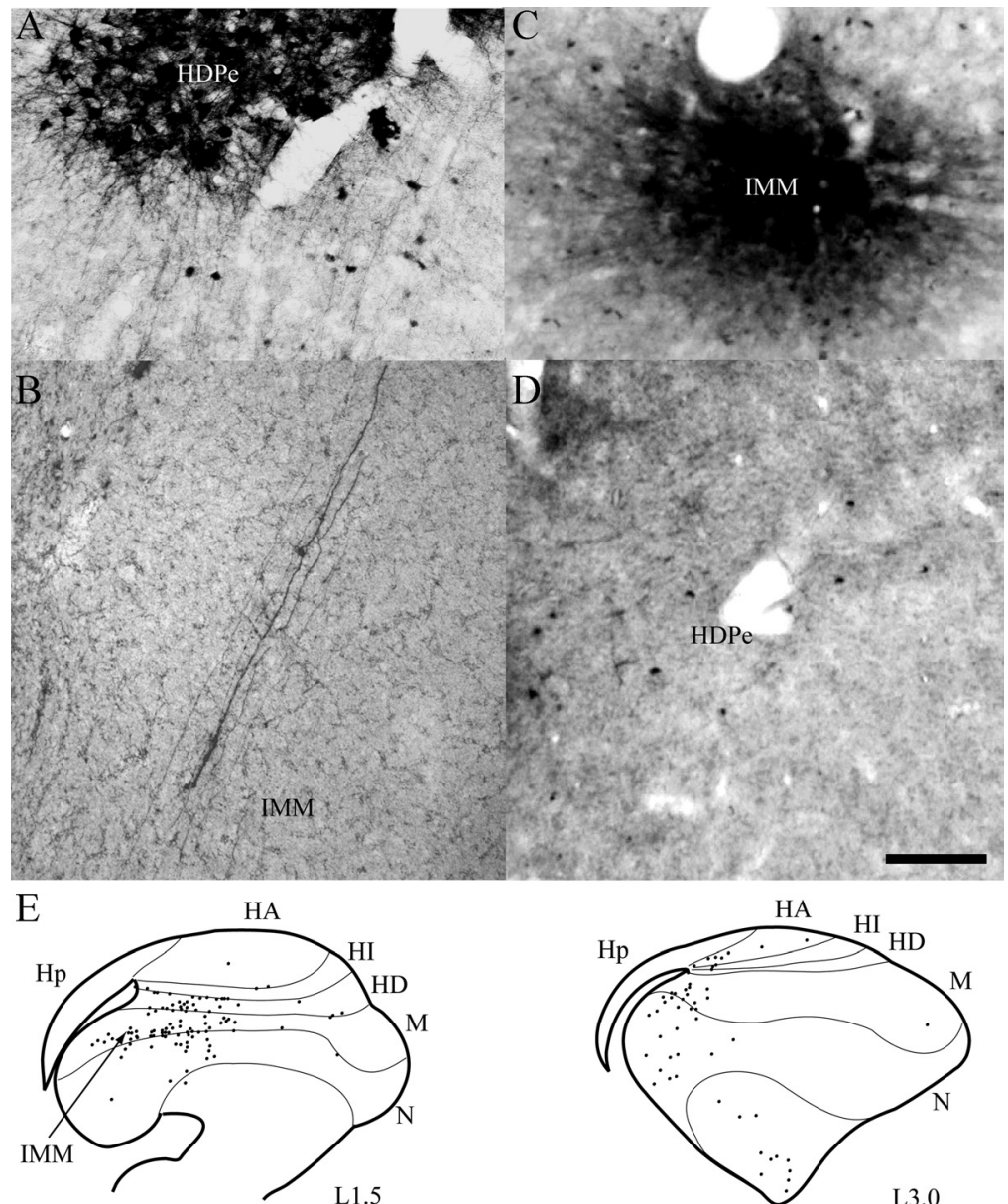

L1.5

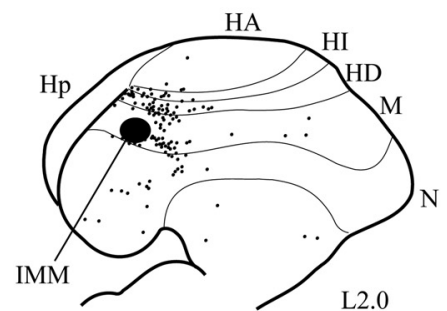

L2.0

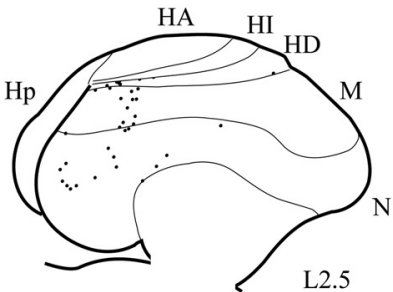

L2.5

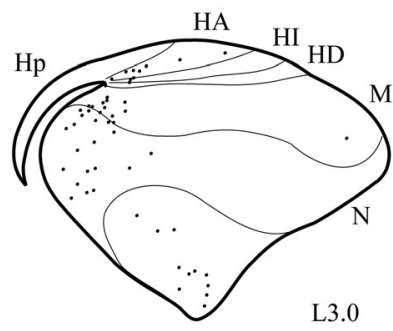

L3.0

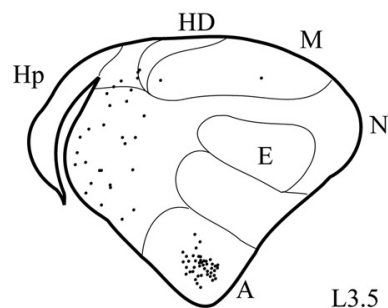

L3.5

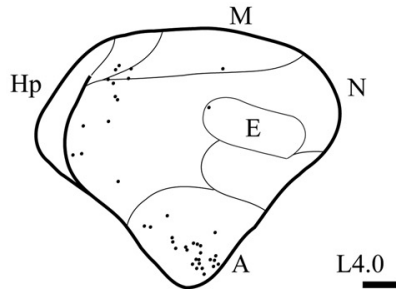

Figure 3. Connections between the HDPe and IMM. $\boldsymbol{A}, \boldsymbol{B}$, Photomicrographs of the BDA injection site in the HDPe $(\boldsymbol{A} ; n=4)$. Projection of the labeled axons to the IMM region $(\boldsymbol{B}) . \boldsymbol{C}, \boldsymbol{D}, A$ CTb injection was made in the $\operatorname{IMM}(\boldsymbol{C} ; n=3)$. Immunoreactive cell bodies are observed in the HDPe region $(\boldsymbol{D})$. $\boldsymbol{E}$, A series of drawings spanning the mediolateral extent of the telencephalon to illustrate the (Tb injection site (solid black) and CTb-immunoreactive cell bodies (dots). Hp, Hippocampus; M, mesopallium; N, nidopallium; A, arcopallium; E, entopallium. Scale bars: $\boldsymbol{D}, 100 \mu \mathrm{m} ; \boldsymbol{E}, 1 \mathrm{~mm}$.

al., 2004). The photodiode currents were amplified, high-pass filtered with a $2.2 \mathrm{~s}$ time constant, and low-pass filtered with a frequency of 1000 $\mathrm{Hz}$. The optical imaging was performed in a still chamber with Ringer's solution at a temperature of $37^{\circ} \mathrm{C}$.

Pharmacological experiment for the optical recording. 6-Cyano-7nitroquinoxaline-2,3-dione (CNQX) (non-NMDA receptor antagonist, $5 \mu \mathrm{M}), \mathrm{APV}(200 \mu \mathrm{M})$, and ifenprodil $(10 \mu \mathrm{M})$ were purchased from Sigma-Aldrich.
Analysis of the optical signals. The signals measured by PDA were presented as the fractional change $(\Delta I / I$, the change in light intensity divided by the direct-current background intensity) calculated by the analysis software Neuroplex (RedShirtImaging), which runs under Interactive Data Language (Research Systems). The 464 signals displayed on the monitor corresponded to the position of each photodiode; therefore, we were able to obtain information on the spatiotemporal pattern of changes in the membrane potential. Signals with amplitudes of signal-to-noise ratio $>3$ were considered to be significant responses evoked by electric stimulation, and the extent of the areas with these signals were regarded as the areas responding to the stimulation. To obtain the velocity of the signal transduction, we divided the total distance of the signal traveled by the time required (see Fig. 5E).

Statistical analysis. All data in this paper are expressed as means \pm SEM. The number of animals used is indicated in each figure or the legends. We used one-way ANOVA, followed by Scheffe's $F$ test to compare the values between conditions (see Figs. 4G, 6B,D). A twoway ANOVA followed by Scheffé's $F$ test was used to compare the number of positive cells between the control and training groups or between brain regions and to compare the reaching distance of signal between experimental groups (see Figs. $5 H, 6 E, 7 F, 8 B, 9 C$ ). The differences between two experimental groups were analyzed using the Student's $t$ test (see Figs. $5 D, E, 7 D, 9 B, 10 C$ ) (supplemental Fig. $\mathrm{S} 4$, available at www.jneurosci.org as supplemental material). To analyze the correlation directly between the PS and total distance traveled for the signal, we used a polynomial regression (see Fig. $6 F$ ). A one-sample $t$ test was used to examine whether or not the PS values differed significantly from chance (0.5) [indicated by \# in Figs. 5F, 6B, $9 B$ and supplemental Fig. S4 (available at www.jneurosci.org as supplemental material)]. Differences were regarded as statistically significant at ${ }^{\star} p<0.05$ and ${ }^{* *} p<0.01$.

\section{Results}

Anatomical identification of the neural circuit from the visual Wulst to the IMM

Neural connections in the VW, excluding the HA

The terminology used to describe structures of the telencephalon is generally based on the nomenclature suggested by Reiner et al. (2004). The IHA, located in the dorsal part of the $\mathrm{VW}$, receives afferent connections from the thalamic nucleus, the DLA (Karten et al., 1973; Watanabe et al., 1983). After injection of the retrograde neural tracer CTb in the IHA at L1.5, labeled neurons were mainly found in the DLA of the thalamus (Fig. $1 A-C$ ), as expected. Then, starting from the IHA, we tried to find the connection between the VW and IMM. The most dorsal part of the VW is the HA, located just dorsal to the IHA. Ventral to the IHA, the HI and HD layers are located in 
the VW. The neural connection of the HA has been scrutinized by Shimizu et al. (1995), and no direct connection with the mesopallium (former HV: hyperstriatum dorsale), including the IMM, was found. Moreover, our optical imaging data did not show any connection between the HA and IMM (data not shown). Therefore, we excluded the HA from our analysis. CTb was injected into several parts of the $\mathrm{HI}$ and $\mathrm{HD}$ in the VW, and, in most cases, the labeled cells were restricted to the region surrounding the injection site and detected in the VW. However, when the $\mathrm{CTb}$ injection was made into the rostral part of the HD (HDR) (0.5-1.5 $\mathrm{mm}$ caudally from the rostral surface and 2.0-3.0 mm ventrally from the dorsal surface) at L1.5, the labeled cells were distributed in the dorsal part of the injection site in the IHA and HA but predominantly in the IHA in all the chicks successfully injected with $\mathrm{CTb}$ in the correct location (Fig. $1 A, D$ ) (supplemental Fig. S1 $A$, available at www.jneurosci.org as supplemental material). The neurons in the HDR were labeled when CTb was injected in the more caudal region of the $\mathrm{HD}$ at L1.75, and this result was confirmed by multiple replications (Fig. $1 A, E-G$ ) (supplemental Fig. S1 $B$, available at www.jneurosci.org as supplemental material). To describe more precisely this ventroposterior part of the HD in the VW, we call this region the HDCo (2.5-3.5 $\mathrm{mm}$ from the rostral surface and $2.5-3.0 \mathrm{~mm}$ from the dorsal surface). When the anterograde tracer BDA was injected in the IHA, HI, or HDR, only short processes were detected and the connections were less evident. Thus, we could not observe any neurons in the IHA or HI in the VW having direct efferent connections with other brain areas.

\section{HDCo neurons project into the HDPe}

We injected BDA in the HDCo at L1.5 and found that long labeled fibers passed through the HD layer in the caudal direction and reached the periventricular region of the HD (Fig. 2A-E). We call this region the periventricular part of the $\mathrm{HD}$ (HDPe). When a DiI crystal was inserted into the HDCo, the bundle of labeled fibers reaching the HDPe was visualized (Fig. $2 \mathrm{~F}-\mathrm{H}$ ). Then, CTb was injected in the HDPe, and retrogradely labeled cell bodies were detected in the more rostral region of the HD, including the HDCo in all samples adequately injected with CTb (Fig. 2I-K,N) (supplemental Fig. S2, available at www.jneurosci.org as supplemental material). This result was also confirmed by DiI labeling of HDPe neurons (Fig. $2 L, M$ ).

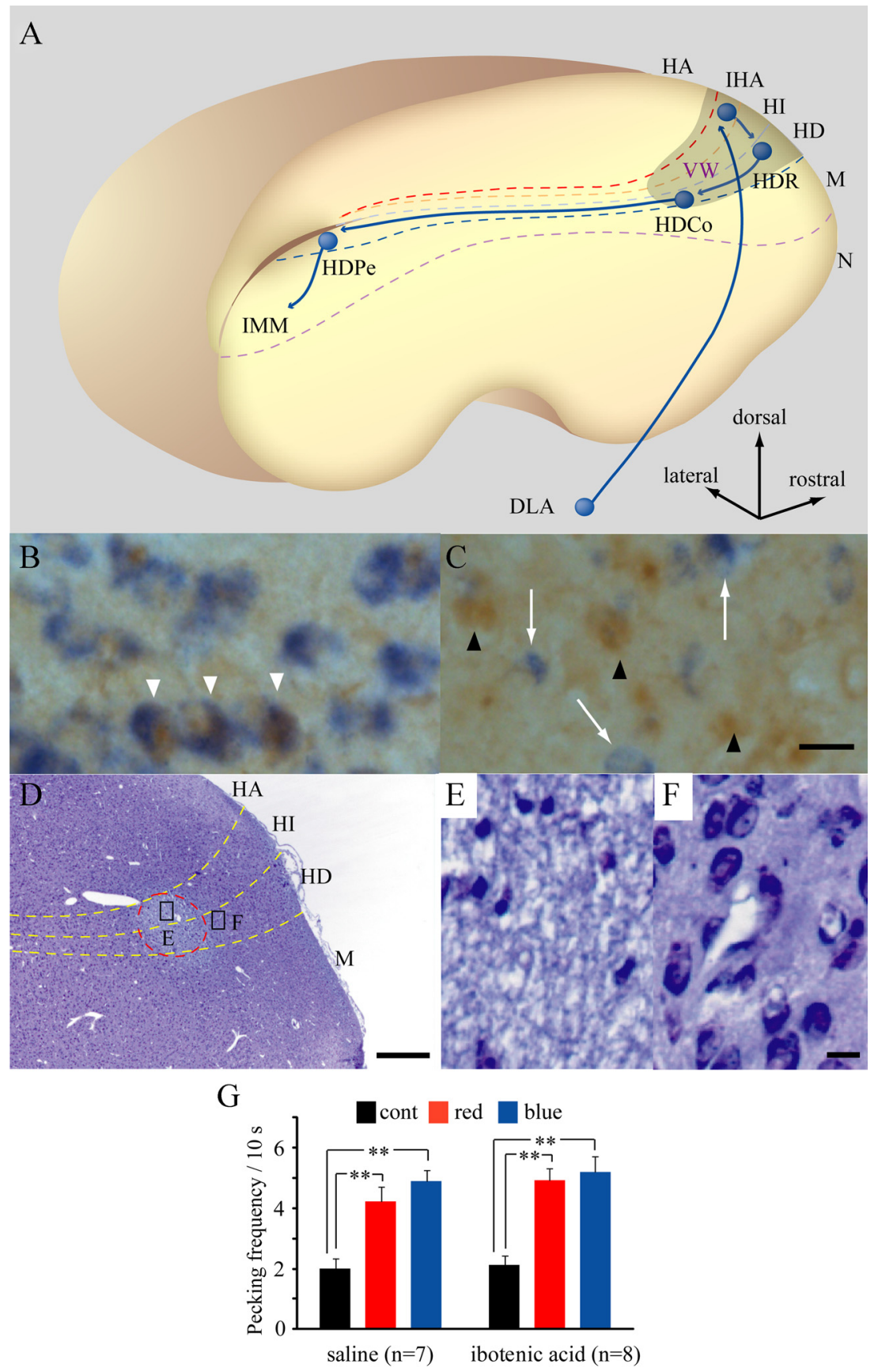

Figure 4. HDCo cells projecting to the HDPe were excitatory cells and indispensable for imprinting behavior. $A$, Schematic drawing of the chick brain (sagittal view) showing the neural circuit from the DLA to the IMM. B, C, CTb was injected into the HDPe, and most of the retrogradely labeled (brown) HDCo cells expressed the excitatory cell marker vGlut2 ( $\boldsymbol{B}$; purple) but not GAD65 ( $\boldsymbol{C}$; purple). White arrowhead, vGlut2-positive (Tb-labeled cell; black arrowhead, CTb single-labeled cell; white arrow, GAD65 single-positive cell. $\boldsymbol{D}$, Nissl-stained sagittal section from a chick injected with ibotenic acid in the $\mathrm{HDC}$. Red circle, Injection site. $\boldsymbol{E}, \boldsymbol{F}$, Enlarged photomicrograph of the region indicated by the boxes in $\boldsymbol{D}$. At the ibotenic acid injection site, selective deletion of the HDCo neurons was observed $(\boldsymbol{E})$. Neurons in the region surrounded by the injection site were not affected $(\boldsymbol{F})$. $\boldsymbol{G}$, Frequency of pecking numbers per $10 \mathrm{~s}$ at the discrimination test was not different between ibotenic acid- and saline-injected groups. ${ }^{* *} p<0.01$. Scale bars: $\boldsymbol{C}, \boldsymbol{F}, 10 \mu \mathrm{m} ; \boldsymbol{D}, 100 \mu \mathrm{m}$.

\section{HDPe neurons send afferent fibers into the IMM}

The HDPe was then injected with BDA, and we found that the labeled fibers extended to the IMM (Fig. $3 A, B$ ). The injection of $\mathrm{CTb}$ into the IMM revealed a great number of retrogradely labeled neurons in the HDPe and in the arcopallium in all samples with successful injection of CTb (Fig. 3C-E) (supplemental Fig. S3, available at www.jneurosci.org as supplemental material). 
A

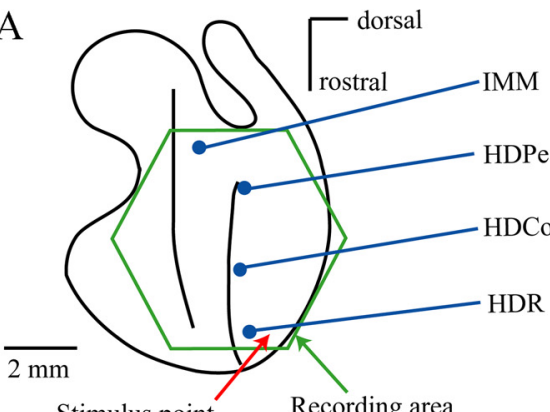

Stimulus point

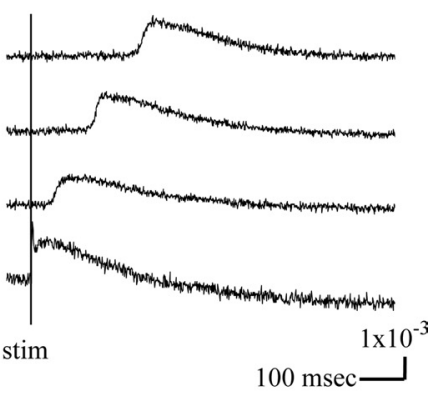

B

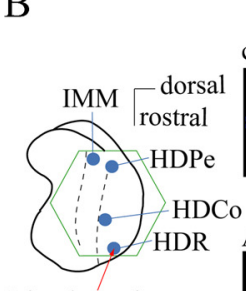

Stimulus point

$$
\text { AP }
$$

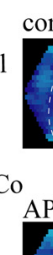

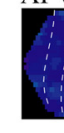

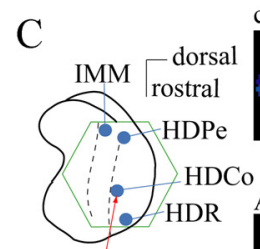

Stimulus point

$\Delta \mathrm{I} / \mathrm{I}$

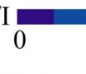

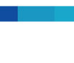

IDC control HDR

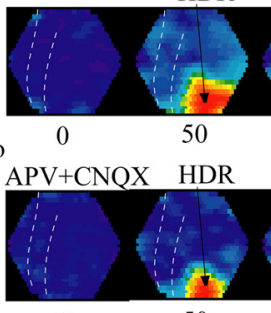

0

50

100

HDCo
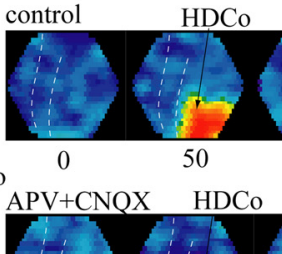

100

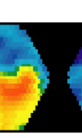

1500

$50 \quad 200$

HDPe

(ms)

HDPe IMM

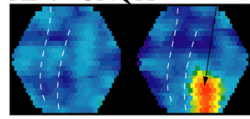

0

50

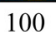

100

150

200

D

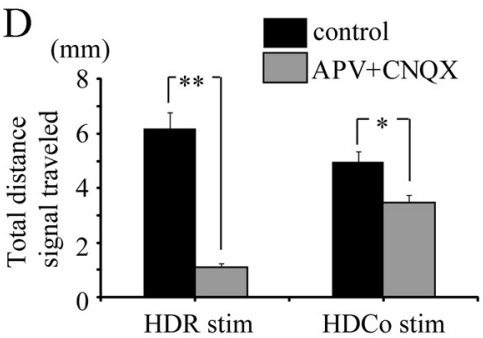

$\mathrm{E}_{(\mathrm{cm} / \mathrm{s})}^{3}$

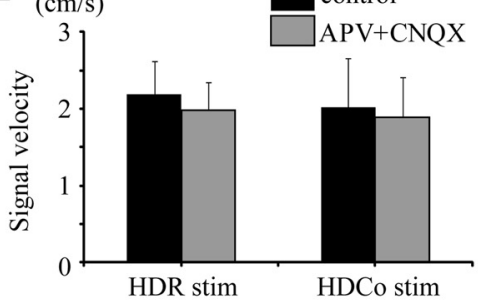

F
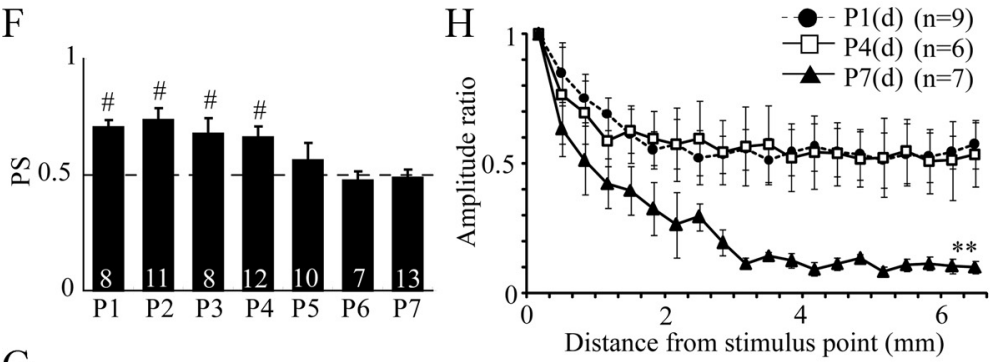

G

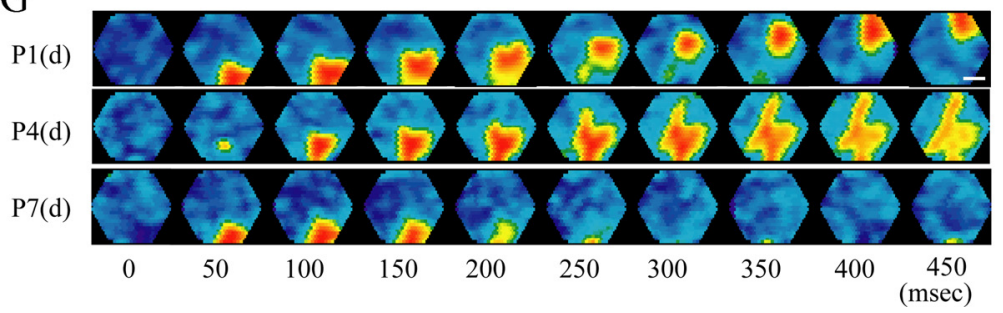

Figure 5. The VW-IMM circuit was visualized by optical imaging, and its activity was high during the critical period for visual imprinting. $\boldsymbol{A}$, Schematic diagram of an acute brain slice used for optical imaging analysis (left) and representative traces of the
The results of these anatomical tracing studies suggest that, in the telencephalon, the visual information is first received by the IHA neurons, then reaches the HDCo neurons in the VW, and finally reaches the IMM neurons via the HDPe neurons (Fig. 4A).

\section{Critical role of the VW-IMM circuit in imprinting}

Ibotenic acid lesions of the HDCo neurons resulted in impaired visual imprinting

To establish the importance of the neural network described above in visual imprinting behavior, we concentrated on the HDCo because it is the most efferent part of the VW that projects to the telencephalic region, the HDPe. First, we examined the expression of the vesicular glutamate transporter and GAD65 mRNA in these HDCo cells and confirmed that the HDCo cells projecting to the HDPe were glutamatergic neurons (Fig. 4B,C). Then, a small lesion was created in the HD area by injecting a small amount of ibotenic acid (Fig. 4D). For analysis, we selected chicks in which only the neurons in the HDCo and the immediate surrounding region were deleted and found that no imprinting behavior was observed in these birds (Fig. $4 D-F$ ) (the PS for saline- or ibotenic acid-treated group are $0.70 \pm 0.042$ and $0.53 \pm 0.048$, respectively; $p<0.05$ ). For chicks in which the lesion was too small or mislocated, the imprinting behavior was not inhibited (supplemental Fig. S4, avail-

$\leftarrow$

optical signal in the HDR, HDCo, HI, HDPe, and IMM evoked by electric stimulation applied to the HDR of P1(d) chicks (right). The recording area is indicated by the hexagonal frame (green). $\boldsymbol{B}$, Spatiotemporal images of signal propagation in P1(d) chicks with stimulation applied in the HDR region (top). The slices were then treated with APV and CNQX, and the responses were similarly recorded (bottom). C, Spatiotemporal images of signal propagation in the $\mathrm{P} 1$ (d) slice with stimulation applied in the HDCo region (top) and the responses after APV and CNQX treatment (bottom). D, Total distance the signal traveled from the stimulus points. Treatment with APV and CNQX inhibited the signal propagation beyond the synapse. $\boldsymbol{E}$, Velocity of the signal transduction. APV and CNQX did not affect the propagation speed. $\boldsymbol{F}$, Dark-reared chicks were trained with a red square at the indicated day after hatching, and the preference for the red square was evaluated $24 \mathrm{~h}$ later. The critical period ends at $\mathrm{P} 4$. \# indicates significantly different from PS $=0.5$. G, Spatiotemporal images of signal propagation evoked by HDR stimulation in the brain slices obtained from chicks of the indicated age. $\boldsymbol{H}$, Relative amplitudes of the signal in the P1(d), P4(d), and P7(d) slices are respectively plotted against the distance from the stimulus point. The HDC 0 and IMM are $\sim 2$ and $5 \mathrm{~mm}$ away from the stimulus point, respectively. Note that the signal was propagated to the IMM during the critical period but not after this period. ${ }^{*} p<0.05$; ${ }^{* *} p<0.01$. Scale bar: $\boldsymbol{B}, \mathbf{G}, 2 \mathrm{~mm}$. 
able at www.jneurosci.org as supplemental material).

We checked the visual ability of the lesioned and control birds. The result of the discrimination test revealed that the chicks of both groups pecked the colored beads at comparable frequencies (Fig. 4G) (saline treated: control, $2.00 \pm 0.33$; red, $4.24 \pm 0.48$; blue, $4.90 \pm 0.35$; for ibotenic acid treated: control, $2.13 \pm 0.31$; red, $4.92 \pm 0.42$; blue, $5.21 \pm 0.51$; difference between the treatments, $F_{(1,39)}=1.08$, $p=0.32)$. This result showed that general vision and visual acuity were maintained in the ibotenic acid-treated chicks.

A functional neural circuit between the $V W$ and IMM in P1 chicks

We then examined the physiological activity of this neural circuit using brain slices. Chicks were hatched and maintained in a quasi-dark incubator. At P1, sagittal brain slices were prepared and subjected to optical imaging of neural activity. When the HDR was electrically stimulated, the signal was transmitted to the IMM via the HDCo and HDPe at a velocity of $2 \mathrm{~cm} / \mathrm{s}$ (Fig. $5 A, B$, top panel). However, when the slice was treated with glutamate receptor blockers APV and CNQX, the signal remained in the VW and was not transmitted beyond the HDCo (Fig. 5B, bottom panel). Stimulation of the HDCo produced a signal that reached the IMM, but treatment with blockers inhibited the propagation of the signal beyond the HDPe (Fig. 5C,D) [HDR stimulation, $6.19 \pm 0.58 \mathrm{~mm}$ (control) and $1.09 \pm 0.15 \mathrm{~mm}$ (APV + CNQX), $p<0.01$; HDCo stimulation, $4.98 \pm 0.37$ $\mathrm{mm}$ (control) and $3.47 \pm 0.29 \mathrm{~mm}$ (APV + CNQX), $p<0.05$ ). However, the velocity of the signal transduction was not significantly different between the control and inhibitors-treated groups (Fig. 5E) (HDR stimulation, $2.19 \pm 0.43 \mathrm{~cm} / \mathrm{s}$ (control) and $1.98 \pm 0.37 \mathrm{~cm} / \mathrm{s}$ (APV + CNQX), $p=0.35$; HDCo stimulation, $2.02 \pm$ $0.64 \mathrm{~cm} / \mathrm{s}$ (control) and $1.89 \pm 0.52$ $\mathrm{cm} / \mathrm{s}(\mathrm{APV}+\mathrm{CNQX}), p=0.42)$. These results suggest that the VW-IMM neural circuit is functional at P1 and that, consistent with the anatomical evidence described above, this signal is mediated transynaptically by neurons bearing glutamate receptors in the HDCo and HDPe.

The activity of this circuit was high during the critical period of visual imprinting but decreased to an undetectable level after the closure of the critical period

We tried to detect changes in signal transduction in this circuit as the critical period progressed. It was therefore necessary to determine the sensitive period for visual imprinting. Chicks were hatched and maintained in a quasi-dark incubator (dark condi-

P7(d)

$\mathrm{P} 7(\mathrm{c})$

P7

(TP1)

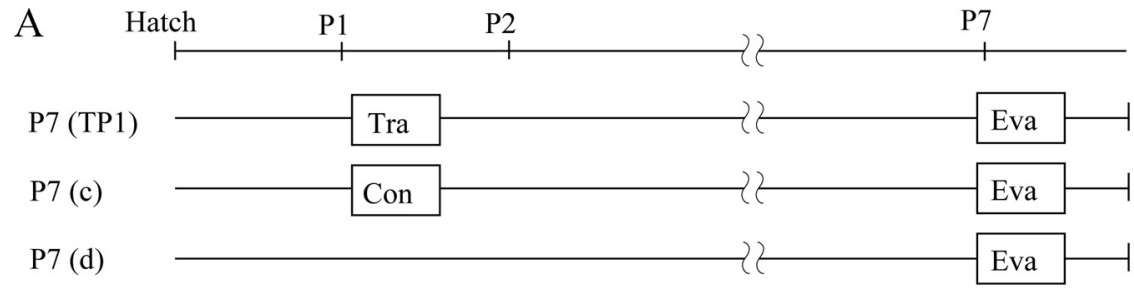

B

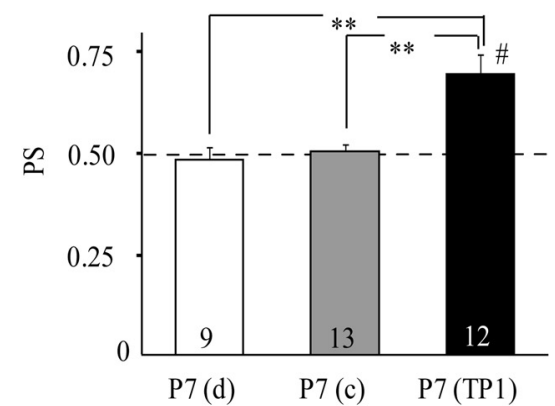

D

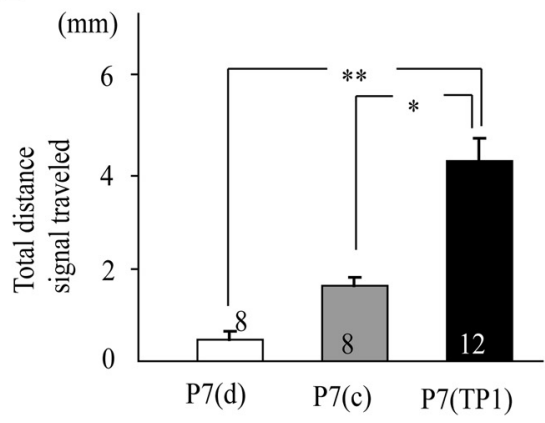

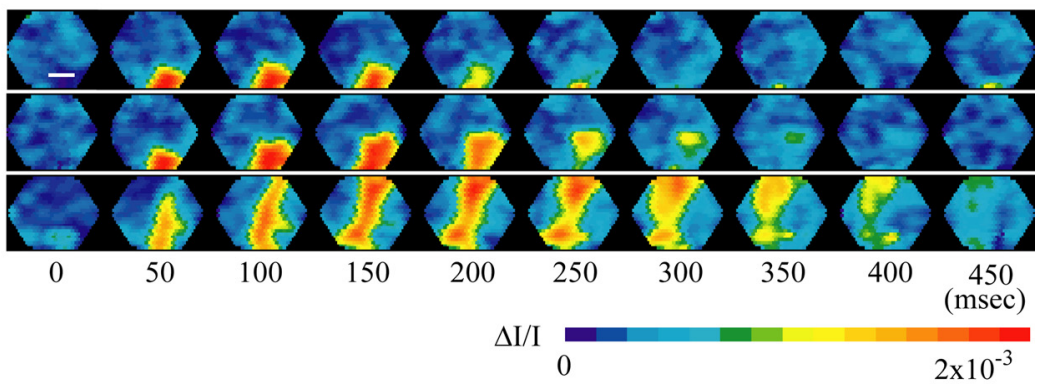
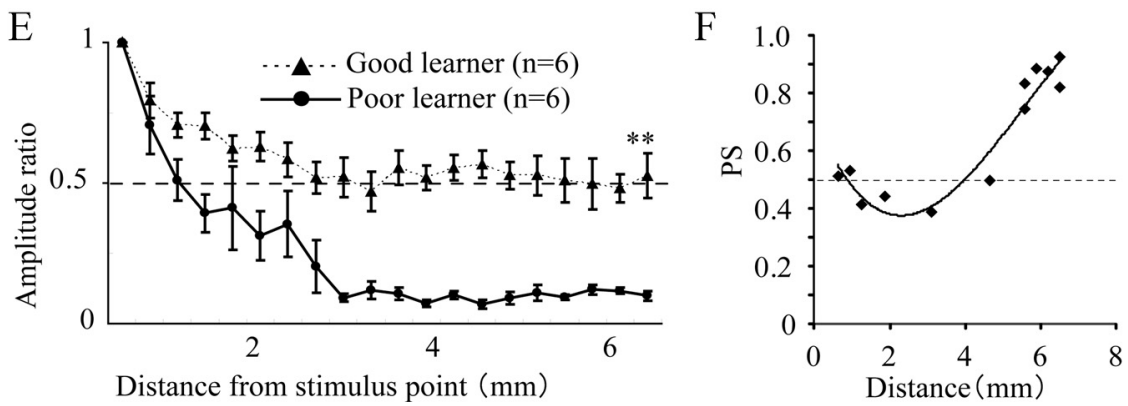

Figure 6. Activity of the VW-IMM pathway was high even at P7 in imprinted chicks. $\boldsymbol{A}$, Experimental schedules. Chicks were trained with a red square presented on the screen (Tra) or exposed to the screen without any image (Con, control training). Eva, Evaluation. $B$, PS at the evaluation for each condition. Only the chicks trained at P1, P7(TP1), showed imprinting behavior at P7. C, Spatiotemporal images of signal propagation evoked by the stimulation of the HDR. D, Furthest point that the signal (signal-to-noise ratio $>3$ ) reached is expressed as the distance from the stimulus point. HDCO and IMM are $\sim 2$ and $5 \mathrm{~mm}$ away from the stimulus point, respectively. The signal propagated to the IMM only in the P7(TP1) chicks. $E$, Relative amplitudes of the signal in the good $(P S \geq 0.65)$ and poor $(P S<0.65)$ learners were respectively plotted against the distance from the stimulus point. In the good learners, the signal reached the IMM but not in the poor learners. $\boldsymbol{F}$, Correlation diagram between the PS and the total distance traveled from the stimulus point. The two factors showed a positive correlation. \# indicates significantly different from PS $=0.5$; ${ }^{*} p<0.05$; ${ }^{* *} p<0.01$. Scale bar, $2 \mathrm{~mm}$.

tion). During the training, they were exposed to a moving red square on the screen for $1 \mathrm{~h}$. The next day, the preference for the red square was determined. When the training was performed between P1 and P4, the PS was significantly higher than chance level (Fig. 5F) (PS for P1, P2, P3, and P4 chicks are $0.71 \pm 0.031$, $0.75 \pm 0.044,0.70 \pm 0.062$, and $0.67 \pm 0.057$, respectively). Thus, the critical period seems to last from P1 to P4 and closes at P5 [PS for P5, P6, and P7: $0.56 \pm 0.067(p=0.12), 0.49 \pm 0.029$ ( $p=$ $0.33)$, and $0.50 \pm 0.02(p=0.48)$, respectively]. Then, sagittal slices of the chick telencephalon were prepared and subjected to 
A

Hatch

P1

P2

$$
\begin{aligned}
& \text { P7(TP1-ctb) } \\
& \text { P7(c-ctb) }
\end{aligned}
$$
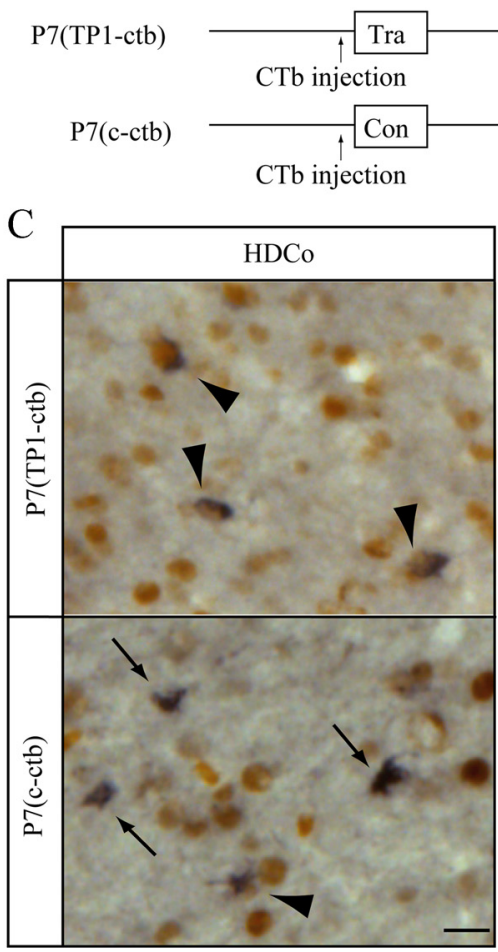

$\mathrm{B}$

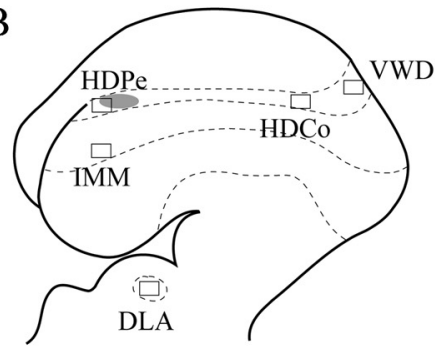

$\mathrm{D}$

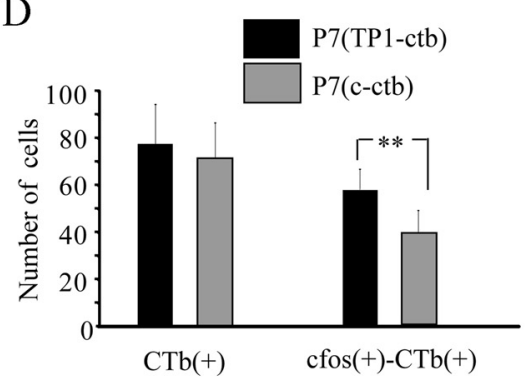

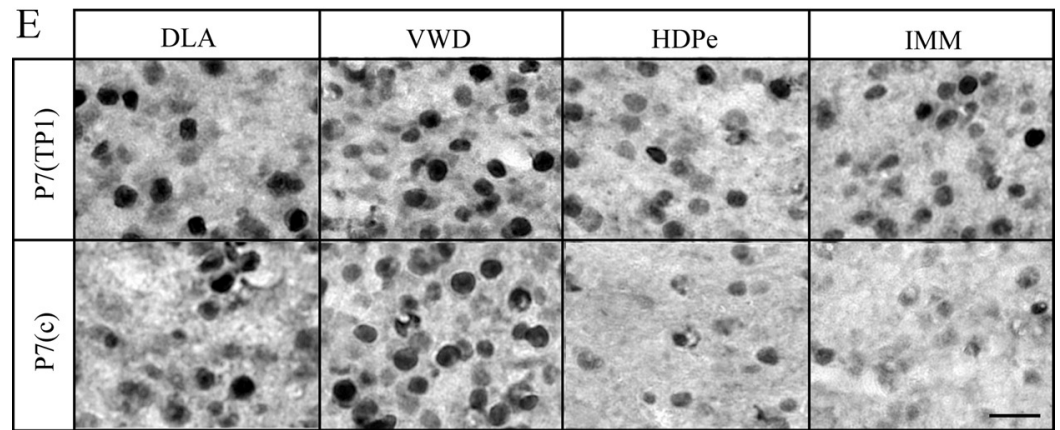

\section{(1)}

$\mathrm{F}$

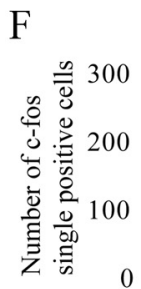

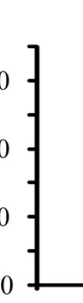

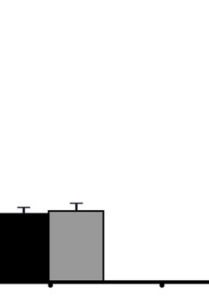

DLA

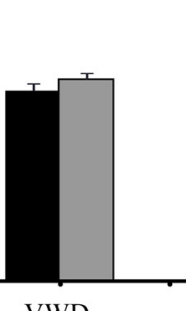

VWD

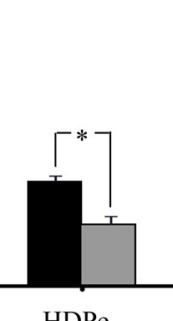

$\mathrm{HDPe}$

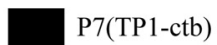

$\square$ P7(c-ctb)

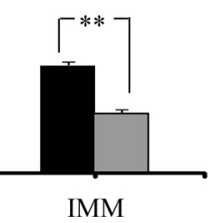

Figure 7. HDCo cells as well as the neurons in the descending pathway in the imprinted chicks could be activated by presenting the imprintstimulus at P7.A, Experimental schedule of $C \mathrm{~Tb}$ injection and behavioral experiment. In the evaluation, only the red square (imprint stimulus) was presented. Fixation was performed $1 \mathrm{~h}$ after the evaluation. $\boldsymbol{B}$, Schema of the sagittal telencephalon, including the VW-IMM pathway. VWD, Dorsal part of the VW. Gray area, CTb injection site. C, Sections presenting the HDCo region from P7(TP1-ctb) and P7(C-ctb) chicks (top and bottom frame, respectively) immunostained with (Tb (black) and CFos (brown). Arrowhead, Double-positive cell; arrow CTbsingle-positive cell. D, Number of (Tb-positivecells and the double-positive cells in the HDCo region of P7(TP1-ctb) and P7(c-ctb) chicks. $E$, Sections from P7(TP1) and P7(C) chick brains showing the expression of cFos in four regions: the DLA, VWD, HDPe, and IMM. F, CFospositive cells were counted in each region ( $n=3$ chicks per group). The number of cFos-positive cells was elevated in the HDPe and IMM, located in the descending pathway from the $H D C 0 .{ }^{*} p<0.05$; ${ }^{* *} p<0.01$. Scale bars: $\boldsymbol{C}, 15 \mu \mathrm{m} ; \boldsymbol{E}, 25 \mu \mathrm{m}$. Tra, Training with a red square presented on the screen; Con, training without any image on the screen; Eva, evaluation.

optical recording. When electric stimulation was applied to the VW of a P1(d) chick slice, the signal was propagated and reached the IMM. At P4, the signal reached the IMM as in the P1 slice. However, the signal did not propagate at P7 (Fig. 5G,H) [differ- ence between $\mathrm{P} 1(\mathrm{~d})$ and $\mathrm{P} 7(\mathrm{~d}), F_{(1,266)}=$ 12.65, $p<0.01$; difference between P4(d) and P7(d), $F_{(1,209)}=19.53, p<0.01$; difference between $\mathrm{P} 1(\mathrm{~d})$ and $\mathrm{P} 4(\mathrm{~d}), F_{(1,247)}=$ $1.34, p=0.32$ ]. This shows that the signal transmission from the VW to the IMM is maintained during the critical period but decreases thereafter.

We checked the neural connection in the P7(d) chick brain by histological methods. When the CTb was injected into the HDPe, the labeled cells were distributed in the HDCo region (supplemental Fig. S5, available at www.jneurosci.org as supplemental material). This result indicates that the fundamental connectivity is maintained even in P7(d) chicks.

\section{The VW-IMM neural circuit remained activated in the P7 chicks trained for imprinting at $P 1$}

We examined and compared the signal transmission in imprinted and nonimprinted chicks. Chicks were hatched and maintained in a quasi-dark incubator (dark condition) (Maekawa et al., 2006, 2007), trained at $\mathrm{P} 1$ by exposure for $1 \mathrm{~h}$ to a red square moving on a screen (TP1), or placed in a training apparatus without seeing any object on the screen (control training) (Fig. 6A). At P7, the preference for the red square was determined. The P7(TP1) chicks had a high PS, but the P7 (c) and the P7(d) chicks failed to show imprinting behavior (Fig. 6B) [PS for P7(d), P7(c), and P7(TP1) chicks are $0.49 \pm 0.035,0.51 \pm 0.019$, and $0.72 \pm$ 0.052 , respectively.] Then, brain slices were prepared, and, when electrical stimulation was applied to the VW, a refined signal transduction linking the VW and IMM via the HD was observed in the P7(TP1) chicks, which showed imprinting behavior, although this response was absent in the P7(c) and P7(d) chicks (Fig. $6 C, D)$ [distances from stimulus point for P7(d), P7(c), and P7(TP1) are $0.48 \pm$ $0.13,192.3 \pm 12.8$, and $432.7 \pm 28.6 \mathrm{~mm}$, respectively]. To examine the correlation between the PS and signal transduction, we divided the P7(TP1) chicks in two groups according to the preference score and compared the signals. In the good learners $(\mathrm{PS} \geq 0.65$; $\mathrm{PS}=0.83 \pm 0.043$, $n=6$ ), the signals were transmitted successfully from the VW to the IMM, but, in the poor learners ( $\mathrm{PS}<0.65$; PS $=0.49 \pm$ $0.072, n=6$ ), the signals did not reach the IMM (Fig. 6E) (difference between the good and poor learners, $F_{(1,190)}=25.35$, $p<0.01)$. We also analyzed the direct correlation between the PS and total distance traveled for the signal and obtained the regression curve given by the following equation: $Y=-0.0055 x^{3}+0.091 x^{2}-$ 
$0.3313 x+0.7238$ (Fig. $6 F)\left(R^{2}=0.9192\right.$, $p<0.01)$. These results reveal the importance of this signaling in imprinting behavior.

HDCo, HDPe, and IMM cells in the chicks trained at $P 1$ were activated when the imprint stimulus was presented at $P 7$

With optical imaging, it is not easy to determine the precise location of the activated cells. To overcome this limitation, we performed histological analysis at the cellular level. CTb was injected in the HDPe before training to enable the identification of the HDCo cells at the time of histological analysis. At P7, only the imprint stimulus was presented, and the chicks were perfused $1 \mathrm{~h}$ after the presentation of this stimulus (Fig. $7 A, B$ ). In this condition, the cells activated at the evaluation expressed an immediate-early gene, cFos, and activated HDCo cells should express cFos antigen as well as CTb (Fig. 7C). Although a comparable number of HDCo cells expressed CTb (Fig. 7D) $[78.2 \pm 16.3$ for P7(TP1-ctb) and 71.1 \pm 18.2 for P7(c-ctb), $p=0.51$ ], the activated cells were more abundant in the trained chicks than in the control-trained chicks $[59.2 \pm 13.7$ for P7(TP1-ctb) and $42.7 \pm$ 14.5 for P7(c-ctb), $p<0.01]$. We also compared the number of cFos-positive cells in other brain areas and found that, in the trained chicks, the number of activated cells was increased in the HDPe (Fig. $7 E, F)(154.7 \pm 7.6$ vs $91.1 \pm 12.5, p<$ $0.05)$ and IMM (159.0 \pm 5.4 vs $88.8 \pm 5.6$, $p<0.01$ ), two regions downstream of the HDCo in the neural circuit. In contrast, in the DLA (101.6 \pm 9.7 vs 105.6 $\pm 11.0, p=$ $0.47)$ and dorsal VW (280.3 \pm 9.9 vs $297.1 \pm 8.4, p=0.39$ ), two areas afferent to the HDCo, training had no effect on the induction of $\mathrm{cFos}$ product.

\section{Role of NMDAR in the induction of imprinting}

The expression of NMDAR in HDCo cells We then focused on the role of the NMDAR in the process of visual imprinting. First, we examined the expression of NMDARs in the HDCo using in situ hybridization. The NMDAR consists of an NR1 subunit and various NR2 subunits. The expression of NR1 in the HDCo increased in the trained chicks compared with that in control-trained chicks, and this change was observed at both $6 \mathrm{~h}[\mathrm{P} 1$; $46.2 \pm 6.5$ (control) and $89.6 \pm 6.3$ (training), $p<0.01]$ (Fig. $8 A, B$ ) and $6 \mathrm{~d}[\mathrm{P} 7 ; 41.8 \pm 7.3$ (control) and $85.2 \pm 5.9$ (training), $p<0.01$ ] after the training. We then examined the subunit composition of NMDAR. In the song bird telencephalon, it is known that the expressions of NR2A and
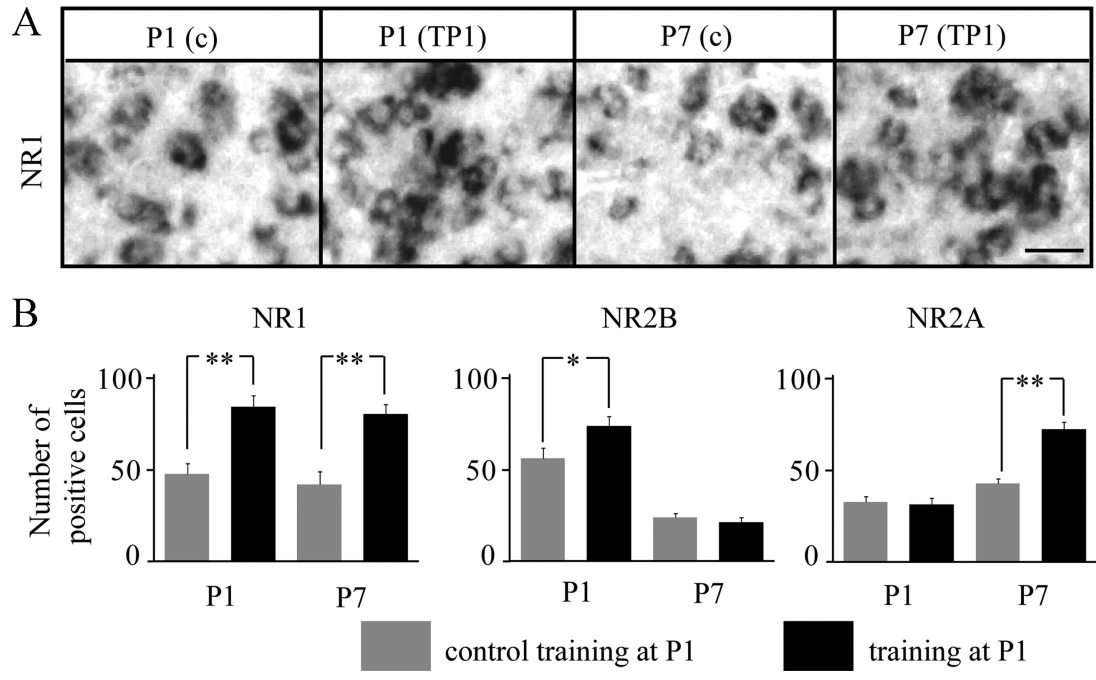

Figure 8. Expression of NMDAR subunits in HDCo cells. $\boldsymbol{A}$, Representative photographs showing induction of NR1 mRNA by imprinting training at P1. B, Comparison of the number of NR1-, NR2B-, and NR2A-positive cells between trained and controltrained chicks in HDC 0 . Increased expressions of the NR1 subunit, NR2B subunit at P1, and NR2A subunit at P7 were observed. $n=$ 3 chicks were used for each group. ${ }^{*} p<0.05$; $^{* *} p<0.01$. Scale bar, $25 \mu \mathrm{m}$.

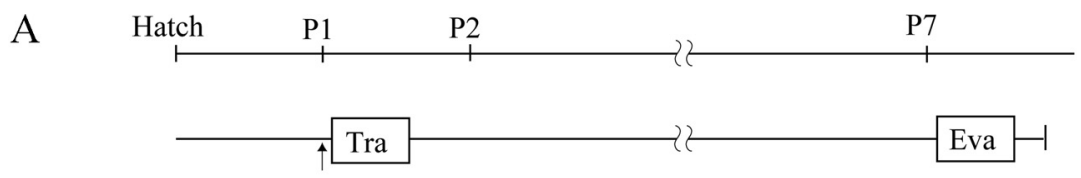

APV, Ifenprodil, or saline injection

$\mathrm{B}$

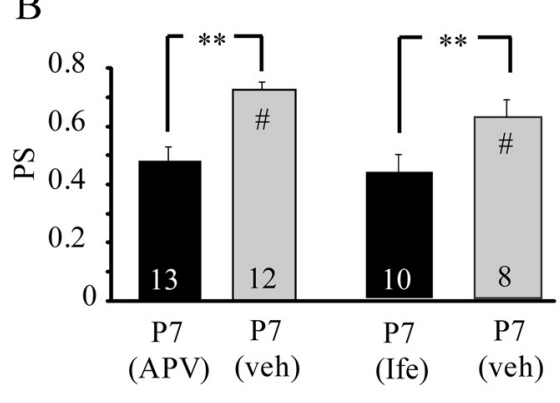

D

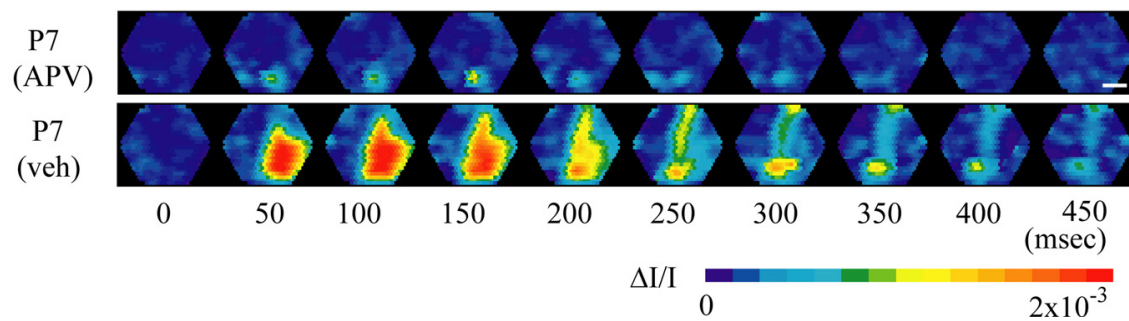

Figure 9. Microinjection of NMDAR antagonists before training at $P 1$ inhibited the imprinting behavior and neural activation in the VW-IMM circuit. $A$, Experimental schedule of microinjection and behavioral experiment. $B, P 7$ chicks injected with APV or ifenprodil into the VW and trained at P1 [P7(APV) or P7(Ife)] did not show imprinting behavior, although those in the control group $[P 7($ veh)] did. C, In the P7(APV) slice, the evoked signal did not propagate. D, Spatiotemporal images of signal propagation in slices from P7(APV) and P7(veh) chicks, respectively. \# indicates significantly different from PS $=0.5 ;{ }^{* *} p<0.01$. Scale bar, $2 \mathrm{~mm}$. Tra, Training with a red square presented on the screen; Eva, evaluation.

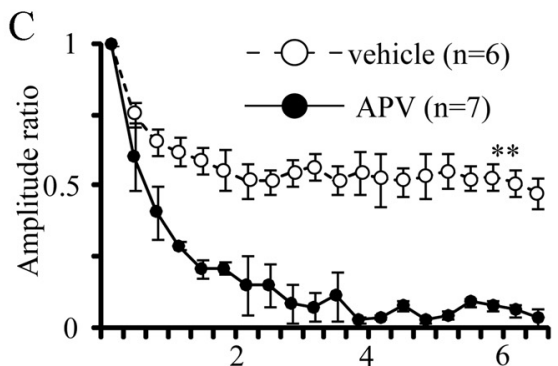

Distance from stimulus point (mm)

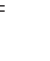




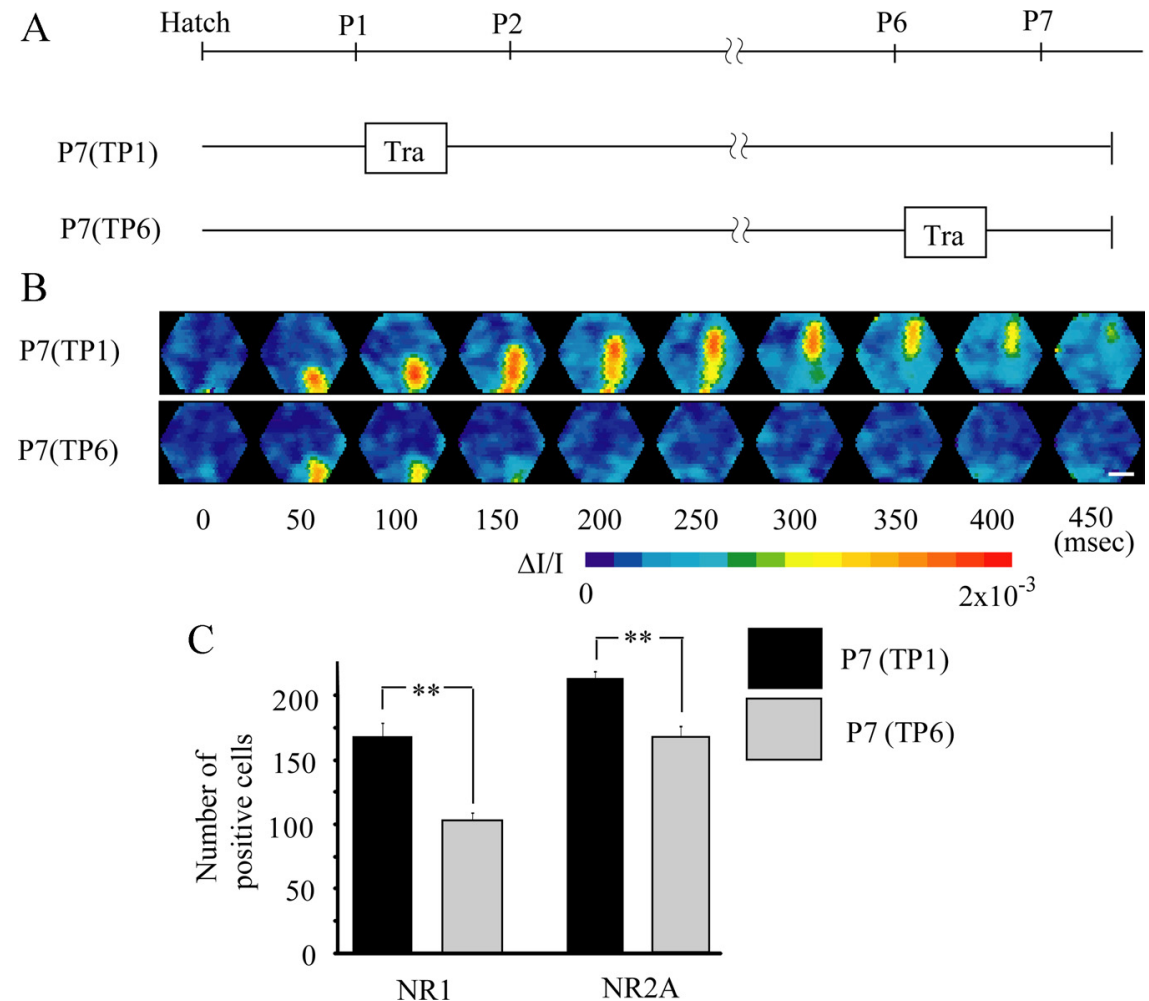

Figure 10. Imprinting training after the closure of the critical period (TP6) could not induce the activation of the VW-IMM neuronal circuit. $\boldsymbol{A}$, Experimental schedule. Chicks were used for physiological or histological experiments at P7, without behavioral evaluation. $\boldsymbol{B}$, Spatiotemporal images of the signal propagation in P7(TP1) and P7(TP6) chicks. During stimulation of the VW, the evoked signal was observed to be transmitted to the IMM in the P7(TP1) slice but not in the P7(TP6) slice. C, Numbers of NR1 mRNA- and NR2A mRNA-expressing cells were lower in the HDC0 of P7(TP6) chicks than in that of P7(TP1) chicks. Sections from three chicks per group were analyzed. ${ }^{* *} p<0.01$. Scale bar, $2 \mathrm{~mm}$. Tra, Training with a red square presented on the screen.

$73.8 \pm 6.8$ (training), $p<0.05]$. The NR2B expression was downregulated during the first few days after hatching, and, at $\mathrm{P} 7$, the NR2A subunit became the major component of the NMDAR in the trained group [39.9 \pm 3.6 (control) and $70.9 \pm 4.7$ (training), $p<0.01]$. These data indicate that the training induced the activation as well as the expression of NMDAR, notably NR2B at the early stage and NR2A at the late stage.

\section{Inhibition of NMDAR in the VW during imprint training} impaired imprint learning

To assess whether or not the activation of NMDARs plays a crucial role in the establishment of imprinting, we examined the effect of the NMDAR antagonist APV or a specific antagonist of the NR2B, ifenprodil (Fig. 9A,B). APV or ifenprodil was applied in the VW at P1, 5 min before the training. At P7, although motor activity was identical in APV- and vehicle-treated chicks (99.7 \pm 17.4 and $112.3 \pm 9.3$ rotations $/ \mathrm{min}$, respectively, $p=0.37$ ) or ifenprodil- and vehicle-treated chicks $(85.2 \pm 13.2$ and $76.6 \pm$ 11.9 rotations/min, respectively, $p=0.58$ ), visual imprinting behavior was suppressed in the APV-treated group $[0.49 \pm 0.06$ for P7(APV) and $0.71 \pm 0.04$ for P7(veh), $p<0.01]$ and in the ifenprodil-treated group [0.47 \pm 0.08 for P7(Ife) and $0.65 \pm 0.07$ for P7(veh), $p<0.01]$. The signal was not transmitted from the VW to the IMM in APV-treated chicks (Fig. 9C,D) (difference between the vehicle- and APV-treated chicks, $F_{(1,247)}=33.79$, $p<0.01)$. Thus, our data suggest that the activation of NMDAR, notably the NR2B subunit in the VW, is a prerequisite for visual imprinting behavior.
Imprint training after the critical period did not induce the activation of the $V W-I M M$ circuit nor the increase in NMDAR expression

When the training was performed between P1 and P4, the PS was significantly greater than chance (Fig. $5 F$ ). Thus, we conclude that the critical period lasts from $\mathrm{P} 1$ to $\mathrm{P} 4$. If the activation and increased expression of NMDAR in this circuit are functionally related to imprinting, these changes should not occur after the training of P6 chicks, in which the critical period is over. With the aim of examining this point, P1 and P6 chicks were trained, evaluated with the trained-in stimulus at P7, and killed 15 min later (Fig. 10A). In the brain slices obtained from the trained P6 chicks [P7(TP6)], no activation of the neural circuit was observed through optical imaging (Fig. 10B). The number of NR1 - or NR2A-positive cells in the HDCo was lower in the P7(TP6) chicks than in the P7(TP1) ones (Fig. 10C) [NR1: $171.3 \pm 10.1$ for $\mathrm{P} 7(\mathrm{TP} 1)$ and $102.6 \pm 7.8$ for P7(TP6), $p<0.01$; NR2A: $212.5 \pm 6.2$ for P7(TP1) and 164.8 \pm 8.9 for P7(TP6), $p<0.01]$. Thus, our data suggest that the increased expression of NMDAR is involved in imprinting behavior.

\section{Discussion}

Our data provide evidence that the neural circuit connecting the VW and IMM is specifically involved in visual imprinting behavior. Signal transduction is facilitated in these regions during the critical period, and the visual imprinting training during this period can enhance and refine this neural circuit. Visual information is processed in the VW, and from the neurons in the HDCo, it is transmitted to the HDPe and then to the IMM. In this context, we found that the HDCo is a key regulatory region for visual imprinting. The HDCo cells are indispensable for imprinting and are activated after visual imprint training. This leads to the refinement and/or enhancement of the signal transduction pathway from the VW to the IMM. After training, the expression of NR2B is increased in the HDCo, and injection of NR2B antagonist into the VW abolished the imprint learning. These data suggest that the NR2B subunit of NMDAR is a key molecule for the activation of HDCo neurons by imprint training.

\section{Neural connection between the VW and IMM}

Previous studies have demonstrated that the IHA in the visual Wulst receives afferent fibers from the DLA in the thalamus (Karten et al., 1973; Watanabe et al., 1983). We confirmed this result by injecting a retrograde tracer into the IHA (Fig. $1 A-C)$.

Our attempt to demonstrate the precise connection in the VW was unsuccessful. This is because neurons with short processes were predominant in the $\mathrm{HI}$ and the rostral part of the HD. Although Shimizu et al. (1995) have demonstrated the projection from the IHA/HD to the HA, other descriptions about the connections in the VW are absent. In contrast, we were able to 
detect $\mathrm{HI}$ neurons that projected to the rostral part of the HD and then to the HDCo (Fig. 1D-G). Therefore, it seems that the HDCo neurons are the output neurons of the VW that transmit the visual information necessary for visual imprinting.

We found that HDCo neurons located in the ventrocaudal region in the VW projected to the HDPe by sending long axons through the HD layer that transversed the telencephalon sagittally (Fig. 2). The HDCo region had not been identified previously, but Shimizu et al. (1995) have reported the existence of anterogradely labeled fibers in the HA that extend to the caudal region, which were observed when an injection was made in the HIS (former nomenclature for HI)/HD. In their Figure 11C, the dorsally located regions of the HV (former nomenclature for mesopallium) may include the HI/HD layer. Therefore, these fibers could correspond to the efferent fibers of the HDCo that we report here. Although Shimizu et al. (1995) have reported the projection from the HI/HD to the hippocampal area, we could not detect labeled fibers projecting to the hippocampal area from the HDCo. This may be attributable to the fact that our injection site was located more medially than in their experiments. Alternatively, the difference in species (pigeon vs chicken) or age (adult vs chick) may lead to different outcomes.

We could not find any description in the literature of the connection between the HDPe and IMM. This may be because the distance between these two regions is no more than $1 \mathrm{~mm}$, and they are therefore not usually studied separately. Contrary to the findings of Shimizu et al. (1995) and our results described above, Bradley et al. (1985) reported a direct connection between the VW and IMM, which they observed during retrograde tracer injection in the IMM. A small amount of tracer may have spread into the HDPe, which could result in cell labeling in the VW.

Concerning the afferent connection to the IMM, Metzger et al. (1998) reported, as a result of retrograde tracing studies, the direct projection from the rostral part of the dorsocaudal nidopallium complex as well as from the mediorostral nidopallium/ hyperpallium ventral. In our study, when the injection was made in the IMM, we also observed retrogradely labeled neurons in the ventral mesopallium and dorsal nidopallium (Fig. 3E, L1.5) as well as in the nidopallium caudolateral to the IMM (Fig. $3 E$, L2.5-3.5). Because the dorsocaudal nidopallium complex has reciprocal connections with the perientopallial belt (Metzger et al., 1998), which receives a projection from the core portion of the entopallium, these connections may serve to integrate the visual information relevant to imprinting from the thalamofugal and tectofugal pathways.

We also confirmed the VW-HDPe-IMM circuit by optical imaging analysis. Synaptic connections were demonstrated in the HDCo and HDPe using blockers for glutamate receptors and by altering the stimulus point (Fig. 5A-E). Therefore, this circuit involving excitatory synapses is already functional at P1.

\section{Role of NR2B in the formation of an enhanced and refined VW-IMM circuit after imprint training}

It has been demonstrated that glutamate release from the IMM is increased in imprinted chicks (Tsukada et al., 1999) and that the inhibition of NMDAR in the IMM or in the dorsocaudal nidopallium impairs imprinting behavior (McCabe et al., 1992; Bock et al., 1997). Besides the role of NMDAR in the IMM and dorsocaudal nidopallium, our data show that the induction of NR2B subunits in the VW, notably in the HDCo, is important for the establishment of imprinting by enhancing the NMDAR activity during imprinting training. In visual imprinting, the neural signal is transmitted to the IMM from the HDCo cells as described in this paper. Therefore, the changes in the IMM neurons may be generated by the presynaptic action of the HDCo cells, in addition to the postsynaptic changes of the IMM cells. It has been reported that NR2B-subunit-containing NMDARs show a longer excitatory postsynaptic potential than receptors comprising NR2A subunits (Monyer et al., 1994); hence, the NR1NR2B complex is considered to be more suitable for detecting synaptic coincidence. During the critical period of imprinting, i.e., from $\mathrm{P} 1$ to $\mathrm{P} 4$, we found that the evoked potential of the VW-IMM circuit is high (Fig. 5). Although the molecular basis of this enhancement is not yet clear, this may reflect the immature state of this circuit. Multiple innervations along this circuit may result in the enhanced activity detected by the optical imaging technique. Alternatively, if the EPSP is higher in the individual HDCo cells of this age, the increase in the intracellular $\mathrm{Ca}^{2+}$ concentration may be enhanced, and this facilitates the induction of long-term potentiation (Malenka and Nicoll, 1993). It will be necessary to test whether longterm synaptic potentiation occurs in the HDCo cells and whether it is facilitated in the P1-P4 HDCo cells during the critical period. Long-term potentiation can regulate dendritic growth and pruning (Engert and Bonhoeffer, 1999; MaleticSavatic et al., 1999; Matsuzaki et al., 2004). In addition, NR2B can function cell autonomously to regulate activitydependent dendrite patterning (Espinosa et al., 2009). Thus, the induction of the NR2B subunit by imprinting training may lead to structural changes in the HDCo cells.

Various experiments in which NMDAR was inactivated by the introduction of antagonist or antisense RNA, or by gene targeting, have shown that NMDAR is implicated in the cellular processes responsible for spatial memory (Morris, 1989; Sakimura et al., 1995; Tsien et al., 1996), cortical map development and plasticity, including those of the barrel (Schlaggar et al., 1993; Iwasato et al., 2000), and visual cortex (Kleinschmidt et al., 1987; Bear et al., 1990; Roberts et al., 1998). Moreover, the developmental and experience-dependent changes in NMDAR subunit expression, as well as the relative contribution of each subunit, have been described in the rodent cortex (Nase et al., 1999; Quinlan et al., 1999; Philpot et al., 2001).

\section{Do the changes in NMDAR subtypes regulate the critical period of visual imprinting?}

The timing of subunit switching from NR2B to NR2A coincided with the closure of the critical period for imprinting, but the role of this switching on the closure of the critical period remains a matter of debate. The end of the barrel cortex critical period is independent of this subunit switching (Lu et al., 2001). In the ferret visual cortex, the subunit switching coincides with the onset, instead of the offset, of the critical period for the ocular dominance column (Roberts and Ramoa, 1999). In contrast, the emerging evidence shows that the GABA-mediated inhibition and regulation of extracellular mechanisms such as proteolysis are important for the plasticity of the critical period of the visual cortex (Hensch et al., 1998; Fagiolini and Hensch, 2000; Mataga et al., 2004).

Our experimental system for chick visual imprinting is a good model for juvenile learning with a well defined critical period. The use of molecular genetic tools now available in chicks (Sato et al., 2007), combined with anatomical and physiological analysis 
at the tissue and cellular levels, will provide useful information concerning the mechanisms responsible for memory formation and plasticity.

\section{References}

Antonini A, Stryker MP (1993) Rapid remodeling of axonal arbors in the visual cortex. Science 260:1819-1821.

Antonini A, Fagiolini M, Stryker MP (1999) Anatomical correlates of functional plasticity in mouse visual cortex. J Neurosci 19:4388-4406.

Bear MF, Kleinschmidt A, Gu QA, Singer W (1990) Disruption of experience-dependent synaptic modifications in striate cortex by infusion of an NMDA receptor antagonist. J Neurosci 10:909-925.

Bock J, Schnabel R, Braun K (1997) Role of the dorso-caudal neostriatum in filial imprinting of the domestic chick: a pharmacological and autoradiographical approach focused on the involvement of NMDA-receptors. Eur J Neurosci 9:1262-1272.

Bradley P, Davies DC, Horn G (1985) Connections of the hyperstriatum ventrale of the domestic chick (Gallus domesticus). J Anat 140:577-589.

Engert F, Bonhoeffer T (1999) Dendritic spine changes associated with hippocampal long-term synaptic plasticity. Nature 399:66-70.

Espinosa JS, Wheeler DG, Tsien RW, Luo L (2009) Uncoupling dendrite growth and patterning: single-cell knockout analysis of NMDA receptor 2B. Neuron 62:205-217.

Fagiolini M, Hensch TK (2000) Inhibitory threshold for critical-period activation in primary visual cortex. Nature 404:183-186.

Gibbs ME, Summers RJ (2005) Contrasting roles for beta1, beta2 and beta3-adrenoceptors in memory formation in the chick. Neuroscience 131:31-42.

Hensch TK, Fagiolini M, Mataga N, Stryker MP, Baekkeskov S, Kash SF (1998) Local GABA circuit control of experience-dependent plasticity in developing visual cortex. Science 282:1504-1508.

Honda Y, Ishizuka N (2004) Organization of connectivity of the rat presubiculum. I. Efferent projections to the medial entorhinal cortex. J Comp Neurol 473:463-484.

Horn G, McCabe BJ, Bateson PP (1979) An autoradiographic study of the chick brain after imprinting. Brain Res 168:361-373.

Iwasato T, Datwani A, Wolf AM, Nishiyama H, Taguchi Y, Tonegawa S, Knöpfel T, Erzurumlu RS, Itohara S (2000) Cortex-restricted disruption of NMDAR1 impairs neuronal patterns in the barrel cortex. Nature 406:726-731.

Kamino K, Hirota A, Fujii S (1981) Localization of pacemaking activity in early embryonic heart monitored using voltage-sensitive dye. Nature 290:595-597.

Karten HJ, Hodos W, Nauta WJ, Revzin AM (1973) Neural connections of the "visual wulst" of the avian telencephalon. Experimental studies in the pigeon (Columba livia) and owl (Speotyto cunicularia). J Comp Neurol 150:253-278.

Kleinschmidt A, Bear MF, Singer W (1987) Blockade of "NMDA" receptors disrupts experience-dependent plasticity of kitten striate cortex. Science 238:355-358.

Kohsaka SI, Takamatsu K, Aoki E, Tsukada Y (1979) Metabolic mapping of chick brain after imprinting using $\left[{ }^{14} \mathrm{C}\right] 2$-deoxyglucose technique. Brain Res 172:539-544.

Lorenz K (1937) The companion in the bird's world. Auk 54:245-273.

Lu HC, Gonzalez E, Crair MC (2001) Barrel cortex critical period plasticity is independent of changes in NMDA receptor subunit composition. Neuron 32:619-634.

Luppi PH, Fort P, Jouvet M (1990) Iontophoretic application of unconjugated cholera toxin $\mathrm{B}$ subunit $(\mathrm{CTb})$ combined with immunohistochemistry of neurochemical substances: a method for transmitter identification of retrogradely labeled neurons. Brain Res 534:209-224.

Maekawa F, Komine O, Sato K, Kanamatsu T, Uchimura M, Tanaka K, OhkiHamazaki H (2006) Imprinting modulates processing of visual information in the visual wulst of chicks. BMC Neurosci 7:75.

Maekawa F, Nakamori T, Uchimura M, Fujiwara K, Yada T, Tsukahara S, Kanamatsu T, Tanaka K, Ohki-Hamazaki H (2007) Activation of cholecystokinin neurons in the dorsal pallium of the telencephalon is indispensable for the acquisition of chick imprinting behavior. J Neurochem 102:1645-1657.

Malenka RC, Nicoll RA (1993) NMDA-receptor-dependent synaptic plasticity: multiple forms and mechanisms. Trends Neurosci 16:521-527.

Maletic-Savatic M, Malinow R, Svoboda K (1999) Rapid dendritic morpho- genesis in CA1 hippocampal dendrites induced by synaptic activity. Science 283:1923-1927.

Mataga N, Mizuguchi Y, Hensch TK (2004) Experience-dependent pruning of dendritic spines in visual cortex by tissue plasminogen activator. Neuron 44:1031-1041.

Matsuzaki M, Honkura N, Ellis-Davies GC, Kasai H (2004) Structural basis of long-term potentiation in single dendritic spines. Nature 429:761-766.

McCabe BJ, Horn G, Bateson PP (1981) Effects of restricted lesions of the chick forebrain on the acquisition of filial preferences during imprinting. Brain Res 205:29-37.

McCabe BJ, Cipolla-Neto J, Horn G, Bateson P (1982) Amnesic effects of bilateral lesions placed in the hyperstriatum ventrale of the chick after imprinting. Exp Brain Res 48:13-21.

McCabe BJ, Davey JE, Horn G (1992) Impairment of learning by localized injection of an $\mathrm{N}$-methyl-D-aspartate receptor antagonist into the hyperstriatum ventrale of the domestic chick. Behav Neurosci 106:947-953.

Metzger M, Jiang S, Braun K (1998) Organization of the dorsocaudal neostriatal complex: a retrograde and anterograde tracing study in the domestic chick with special emphasis on pathways relevant to imprinting. J Comp Neurol 395:380-404.

Miyakawa N, Yazawa I, Sasaki S, Momose-Sato Y, Sato K (2003) Optical analysis of acute spontaneous epileptiform discharges in the in vivo rat cerebral cortex. Neuroimage 18:622-632.

Momose-Sato Y, Honda Y, Sasaki H, Sato K (2004) Optical mapping of the functional organization of the rat trigeminal nucleus: initial expression and spatiotemporal dynamics of sensory information transfer during embryogenesis. J Neurosci 24:1366-1376.

Monyer H, Burnashev N, Laurie DJ, Sakmann B, Seeburg PH (1994) Developmental and regional expression in the rat brain and functional properties of four NMDA receptors. Neuron 12:529-540.

Morris RG (1989) Synaptic plasticity and learning: selective impairment of learning rats and blockade of long-term potentiation in vivo by the $\mathrm{N}$-methyl-D-aspartate receptor antagonist AP5. J Neurosci 9:3040-3057.

Nase G, Weishaupt J, Stern P, Singer W, Monyer H (1999) Genetic and epigenetic regulation of NMDA receptor expression in the rat visual cortex. Eur J Neurosci 11:4320-4326.

Obaid AL, Orkand RK, Gainer H, Salzberg BM (1985) Active calcium responses recorded optically from nerve terminals of the frog neurohypophysis. J Gen Physiol 85:481-489.

Orbach HS, Cohen LB, Grinvald A (1985) Optical mapping of electrical activity in rat somatosensory and visual cortex. J Neurosci 5:1886-1895.

Philpot BD, Sekhar AK, Shouval HZ, Bear MF (2001) Visual experience and deprivation bidirectionally modify the composition and function of NMDA receptors in visual cortex. Neuron 29:157-169.

Quinlan EM, Philpot BD, Huganir RL, Bear MF (1999) Rapid, experiencedependent expression of synaptic NMDA receptors in visual cortex in vivo. Nat Neurosci 2:352-357.

Reiner A, Perkel DJ, Bruce LL, Butler AB, Csillag A, Kuenzel W, Medina L, Paxinos G, Shimizu T, Striedter G, Wild M, Ball GF, Durand S, Güntürkün O, Lee DW, Mello CV, Powers A, White SA, Hough G, Kubikova L, Smulders TV, Wada K, Dugas-Ford J, Husband S, Yamamoto K, Yu J, Siang C, Jarvis ED, Gütürkün O (2004) Revised nomenclature for avian telencephalon and some related brainstem nuclei. J Comp Neurol 473:377-414.

Roberts EB, Ramoa AS (1999) Enhanced NR2A subunit expression and decreased NMDA receptor decay time at the onset of ocular dominance plasticity in the ferret. J Neurophysiol 81:2587-2591.

Roberts EB, Meredith MA, Ramoa AS (1998) Suppression of NMDA receptor function using antisense DNA block ocular dominance plasticity while preserving visual responses. J Neurophysiol 80:1021-1032.

Sakimura K, Kutsuwada T, Ito I, Manabe T, Takayama C, Kushiya E, Yagi T, Aizawa S, Inoue Y, Sugiyama H, Mishina M (1995) Reduced hippocampal LTP and spatial learning in mice lacking NMDA receptor epsilon 1 subunit. Nature 373:151-155.

Salzberg BM, Obaid AL, Senseman DM, Gainer H (1983) Optical recording of action potentials from vertebrate nerve terminals using potentiometric probes provides evidence for sodium and calcium components. Nature 306:36-40.

Sato Y, Kasai T, Nakagawa S, Tanabe K, Watanabe T, Kawakami K, Takahashi Y (2007) Stable integration and conditional expression of electroporated transgenes in chicken embryos. Dev Biol 305:616-624. 
Schlaggar BL, Fox K, O’Leary DD (1993) Postsynaptic control of plasticity in developing somatosensory cortex. Nature 364:623-626.

Shibata H, Kondo S, Naito J (2004) Organization of retrosplenial cortical projections to the anterior cingulate, motor, and prefrontal cortices in the rat. Neurosci Res 49:1-11.

Shimizu T, Cox K, Karten HJ (1995) Intratelencephalic projections of the visual wulst in pigeons (Columba livia). J Comp Neurol 359:551-572.

Sugihara I, Shinoda Y (2004) Molecular, topographic, and functional organization of the cerebellar cortex: a study with combined aldolase $\mathrm{C}$ and olivocerebellar labeling. J Neurosci 24:8771-8785.

Tsien JZ, Huerta PT, Tonegawa S (1996) The essential role of hippocampal CA1 NMDA receptor-dependent synaptic plasticity in spatial memory. Cell 87:1327-1338.

Tsukada Y, Kanamatsu T, Takahara H (1999) Neurotransmitter release from the medial hyperstriatum ventrale of the chick forebrain accompa- nying filial imprinting behavior, measured by in vivo microdialysis. Neurochem Res 24:315-320.

Van der Loos H, Woolsey TA (1973) Somatosensory cortex: structural alterations following early injury to sense organs. Science 179:395-398.

Wada K, Sakaguchi H, Jarvis ED, Hagiwara M (2004) Differential expression of glutamate receptors in avian neural pathways for learned vocalization. J Comp Neurol 476:44-64.

Watanabe M, Ito H, Masai H (1983) Cytoarchitecture and visual receptive neurons in the Wulst of the Japanese quail (Coturnix coturnix japonica). J Comp Neurol 213:188-198.

Wiesel TN, Hubel DH (1965) Comparison of the effects of unilateral and bilateral eye closure on cortical unit responses in kittens. J Neurophysiol 28:1029-1040.

Woolsey TA, Wann JR (1976) Areal changes in mouse cortical barrels following vibrissal damage at different postnatal ages. J Comp Neurol 170: 53-66. 